\title{
Modelling the effect of freestream turbulence on dynamic stall of wind turbine blades
}

\author{
Yusik Kim*, Zheng-Tong Xie** \\ Faculty of Engineering and the Environment,University of Southampton, SO17 1BJ, \\ Southampton, UK
}

\begin{abstract}
Large-eddy simulations of flow over a pitching airfoil are conducted to study the effect of freestream turbulence on the aerodynamic characteristics. A primary field of applications of this study is wind turbine aerodynamics. The wind turbines operate in yaw in large scale variations of wind direction due to very large turbulence eddies, and the blades operate in a periodically oscillating condition. The pitching frequency of the airfoil corresponds to a typical rotating frequency of modern large wind turbines. A divergence-free synthetic turbulence inflow is applied at the upstream region of the pitching airfoil to investigate the effect of small-scale freestream turbulence on dynamic stall. Phase-averaged lift, drag and moment of the pitching airfoil show good agreement with experimental data in the literature. Characteristic phenomena of dynamic stall, such as leading edge vortex motions, are analysed and quantified. The effect of the small-scale upstream turbulence is significant on the lift coefficient during the downstroke. The power spectral
\end{abstract}

\footnotetext{
${ }^{*}$ Current address: University of Stuttgart, IAG, Pfaffenwaldring 21, 70569 Stuttgart, Germany

** Corresponding author: Tel +44(0)23 80594493

Email address: z.xie@soton.ac.uk (Zheng-Tong Xie )
} 
density of the streamwise velocity sampled from one point in the wake shows that the inertial sub-range tends to extend towards the pitching mode for the turbulent inflow, while there is a distinctive spectral gap for the laminar inflow.

Keywords: Dynamic stall, large-eddy simulation, wind turbine aerodynamics, freestream turbulence

\section{Introduction}

Wind turbines operate in turbulent atmospheric boundary layers. It is 3 of great interest to understand the effects of turbulence on the aerodynamic 4 characteristics. There are two reasons for this: (1) wind turbines operate 5 in yaw in large scale variations of wind direction (e.g. much greater than 6 the diameter of the wind turbine disk due to very large turbulence eddies 7 and meso-scale variations), and the blades operate in a periodically oscil8 lating condition and dynamic stall occurs frequently; (2) upstream small 9 turbulence eddies (i.e. comparable to the blade chord length) may affect separation and reattachement on the turbine blade even in relatively steady 11 winds (i.e. quasi-steady conditions). Usually wind turbines operate in a 12 combined condition of (1) and (2), which may significantly affect wind their reducing their expected service life.

15 Existing dynamic stall models do not consider the effect of upstream small scale turbulence or simply use static airfoil data measured in wind tunnels with upstream turbulence. Such approaches ignore important dynamics of 18 the boundary layer over turbine blades during the dynamic stall process. 
It is crucial to understand the effect of freestream turbulence on dynamic stall. There is little thus far reported in the literature regarding the effect of the small-scale freestream turbulence on dynamic stall. Amandolèse and Széchényi (2004) [1] experimentally tested the effects of the mean angle of attack, reduced frequency, pitching amplitude and turbulence intensity on dynamic stall of a pitching airfoil. They reported that the lift overshoot and the time delay of the maximum lift in dynamic stall increased as the turbulence intensity increased. To our knowledge, there is no numerical simulation work reported on this point, and our paper is focused on this. Before turning to the methodology of modelling the effects of small-scale freestream turbulence, it would be worth reviewing characteristic phenomena of dynamic stall in a 'smooth' (laminar) inflow, which can serve as baseline features.

Dynamic stall is a phenomenon associated with an unsteady airfoil motion that presents large hysteresis on the lift and pitching moments while the time varying incidence is beyond its static stall angle [2]. For a pitching airfoil, stall occurs at a higher angle of attack than that for a static one. Also the behaviour of lift and moment coefficients suggests a large hysteresis with respect to angle of attack [3]. Considering the wind turbine aerodynamics, yawed wind (when wind is not normal to the rotating plane of a turbine), wind shear, tower shadow, wake from the upstream turbine, gusts and large atmospheric turbulence eddies, all contribute to unsteady inflow conditions which can lead to dynamic stall.

Some experimental studies have been conducted studying dynamic stall. Carr et al. (1977) [2] reported that virtually all airfoils experience a fully 
developed dynamic stall. They concluded that airfoil geometry, frequency of pitching, amplitude of pitching and Reynolds number were the parameters affecting on dynamic stall in order of decreasing importance. They also reported that locations of the flow reversal and flow separation were distinctively different on a pitching airfoil, while they occurred at almost the same point on a static airfoil.

For the purpose of helicopter rotor design, extensive studies on dynamic stall of oscillating airfoils have been performed $[2,4,3,5]$. Their interests were, however, on relatively small amplitudes of oscillation and small mean angles of attack because helicopters are designed to avoid deep stall conditions [6].

In wind turbine aerodynamics, dynamic stall can be characterized by the rotating frequency of wind turbines. When the upstream wind is not normal to the rotating plane, the sectional blade operates in a periodically oscillating condition at the frequency of the turbine rotation. Note again that dynamic stall can also occur due to a dynamic inflow and atmospheric turbulence etc.

Considering the relation between the period (time unit) for the rotation and the time scale for the flow passing over blade sections leads to the socalled reduced frequency,

$$
k_{\mathrm{red}}=\frac{\omega c}{2 U_{\infty}}
$$

63 where $\omega$ is the pitching frequency, $c$ is the chord length and $U_{\infty}$ is the freestream velocity. In wind turbine aerodynamics, $U_{\infty}$ is a function of the upstream velocity, the rotating frequency of the blade and the radius distance from the hub; $\omega$ is characterized by the rotating frequency of the blade. A 
considerable number of works have been conducted on dynamic stall for variable reduced frequencies on pitching airfoils $[2,7,3,5,8]$ and rotating blades $[6,9,10]$. Previous studies are summarized in Table 1 , where the effective velocity, $U_{\text {eff }}$ is used instead of $U_{\infty}$ in Eq. 1 to estimate the reduced frequency $k_{\text {red. }} U_{\text {eff }}$ is defined as,

$$
U_{\text {eff }}=\sqrt{\left(U_{\infty}\right)^{2}+(r \omega)^{2}} .
$$

Modern large wind turbines have a blade diameter greater than $100 \mathrm{~m}$ and the cut-in and cut-out wind speeds are generally $5 \mathrm{~m} / \mathrm{s}$ and $25 \mathrm{~m} / \mathrm{s}$ respectively. With these conditions, the reduced frequencies of a modern large wind turbine are mostly placed within the range in which dynamic stall is reported in the literature (see Table 1). Therefore, dynamic stall does occur on wind turbines.

Dynamic stall on a pitching airfoil has been being investigated by solving the Reynolds Averaged Navier-Stokes (RANS) equations ever since the computer power allowed it. Ekaterinaris and Menter (1994) [11], Barakos and Drikakis (2000) [12] and Wang et al. (2010) [13] presented numerical studies on dynamic stall and showed agreement with the measurements for some cases. Ekaterinaris and Platzer (1997) [14] presented a comprehensive review on the prediction methods for dynamic stall. They pointed out that the RANS approaches were not reliable to predict the aerodynamic hysteresis for complex flows, such as flow reattachment during the downstroke and deep stall. Wang et al.(2010) [13] confirmed that 2-D RANS models were limited for modelling fully detached flows, e.g. at a high angle of attack. They suggested that advanced CFD methods such as large-eddy simulations 
or detached-eddy simulations had to be adopted to capture the hysteresis. For a rigorous understanding for stall delay on a pitching airfoil, unsteady boundary layers have to be well understood but this has not yet been accomplished [15].

Nagarajan [16] conducted a comparison of RANS and LES for prediction of sound generated by a pitching airfoil at a transitional Reynolds number. They concluded that some crucial features were missing in the RANS results. Haase [17] used both Unsteady Reynolds-Average Navier-Stokes (URANS) simulations and Detached Eddy Simulations(DES) to simulate the dynamic stall of a pitching airfoil at Reynolds number $9.8 \times 10^{5}$. They noticed that the latter performs better.

This paper focuses on two points. Firstly, an advanced approach, i.e. LES, is adopted to provide a reliable prediction for dynamic loads on wind turbines. Though the effect of freestream turbulence on dynamic stall is a primary subject on this study, we dedicated a substantial part (Sections 3 and 4) on dynamic stall with a 'smooth' (laminar) inflow. This is because it would provide reference data for the turbulent inflow cases. In addition, simulating unsteady flows over a pitching airfoil using LES is a relatively new and very challenging task, and thus it is of great interest to explore further.

Secondly, the effect of freestream turbulence on dynamic stall characteristics is investigated using LES. The methodology is summarized in Section 2. In Section 3, a pitching NACA 0012 airfoil is simulated and the results are validated against experimental data $[7,18]$ to provide a baseline simulation. Then, significant features of dynamic stall such as stall delay and leading edge vortices (LEV) are characterized by the aerodynamic force coefficients 
and flow visualizations in Section 4. The effect of freestream turbulence on the flow over a pitching airfoil is investigated in Section 5. A summary and concluding remarks are presented in Section 6.

\section{Methodology}

The Reynolds number is $R e=135,000$ based on the chord $c$ and freestream velocity $U_{\infty}$. The pitching motion is described using the angle of attack,

$$
\alpha(t)=10^{\circ}+15^{\circ} \sin (\omega t)
$$

The reduced frequency $k_{\text {red }}$ ranges from 0.025 to 0.1 in this study. The pitching axis is at the quarter chord point from the leading edge.

The Reynolds number used $(R e=135,000)$ for the current study is in the same order of magnitude as those for medium and small size wind turbines, but is at least one order of magnitude lower than those for the large wind turbines. It is arguable whether the data at the current Reynolds number can be extrapolated to represent the flow at a higher Reynolds number, e.g. $O\left(10^{6}\right)$. Rinoie and Takemura [18] reported that the size of the separation bubble was less than 0.1 chord length with the same airfoil at the same Reynolds number, and most of the boundary layer was fully turbulent. Lissaman [19] showed that $R e \sim 70,000$ was a critical number classifying low and high Reynolds number, which is far below the Reynolds number $R e=135,000$ used in this paper. It is to be noted that an aerofoil subjected to freestream turbulence is less Reynolds number dependent than that subjected to a smooth flow. Therefore, the current study will be very useful for research and design of medium and small size wind turbines, and can be extrapolated for larger wind turbines. 


\subsection{Dynamic mesh}

The pitching motion of the airfoil was pre-defined and the dynamic mesh approach was adopted for the mesh in the near-airfoil region to accommodate the deformation of the domain due to the airfoil motion. The "dynamic mesh" refers to changing the relative distance between grid points (i.e. squeezing and stretching cells) in time to adjust to an unsteady motion of the subject. The pimpleDyMFoam solver in OpenFOAM was used to deal with this. The conservation equation of property $\phi$ over an arbitrary moving 
control volume $V_{C}$ in integral form is,

$$
\frac{d}{d t} \int_{V_{C}} \phi d V_{C}+\int_{A} d \boldsymbol{A} \cdot\left(\boldsymbol{u}-\boldsymbol{u}_{b}\right) \phi=\int_{V_{C}} \boldsymbol{\nabla} \cdot(\Gamma \boldsymbol{\nabla} \phi) d V_{C},
$$

where $\boldsymbol{u}$ is the fluid velocity vector, $\boldsymbol{A}$ is the outward pointing surface area vector and $\boldsymbol{u}_{b}$ is the boundary velocity vector of the cell-face. Note that $\Gamma$ is the diffusivity coefficient. The local boundary velocity, $\boldsymbol{u}_{b}$, is interpolated from the point velocity, $\boldsymbol{u}_{p}$, which is imposed at each vertex of the control volume. To govern the vertex motion, the Laplacian operator with a diffusivity, $\gamma$, is adopted [24],

$$
\boldsymbol{\nabla} \cdot\left(\gamma \boldsymbol{\nabla} \boldsymbol{u}_{p}\right)=0
$$

The boundary conditions for Eq. 5 are calculated from the known boundary motion, e.g. a moving wall. Then the vertex position at the time level $n+1$ is updated using $\boldsymbol{u}_{p}$,

$$
\boldsymbol{x}^{n+1}=\boldsymbol{x}^{n}+\boldsymbol{u}_{p} \Delta t
$$
Several types of the diffusivity were examined by [24] such as,

$$
\begin{aligned}
& \gamma=\text { const., } \quad \text { constant; } \\
& \gamma=\frac{1}{l}, \quad \text { linear; } \\
& \gamma=\frac{1}{l^{2}}, \quad \text { quadratic; } \\
& \gamma=e^{-l}, \quad \text { exponential, }
\end{aligned}
$$


where $l$ is the cell centre distance to the nearest selected boundary. Jasak and Tuković (2004) [24] investigated the effect of the diffusivity on the mesh quality at the trailing edge of the moving airfoil. They found that the mesh quality is superior using the quadratic diffusivity to that using the constant diffusivity. Thus the quadratic diffusivity was adopted for the current study. As all grid point motion is governed by Eq. 6, an explicit interface between the static and dynamic mesh region is not required.

\section{Baseline simulations}

Typical airfoils used in horizontal axis wind turbines (HAWT) are thick and cambered. However, it is noted that the effect of airfoil shape does not seem to be dominant over the pitching motion for dynamic stall [5]. Carr et al. [2] also support this argument: the hysteresis for the NACA 0012 and chambered airfoil are very similar. These remarks might not be surprising as at a high angle of attack the air flow 'feels' the airfoil as a bluff body. In this regards, we expect that the characteristic behaviours of dynamic stall will be similar to those for typical wind turbine profiles. The 2 nd reason to use NACA 0012 in this paper is that rich and reliable experimental data are available for validation.

Using LES for a number of cycles of pitching motion of an airfoil is timeconsuming and extremely expensive in terms of computation, even though the UK National Supercomputing Service HECToR was used. In order to get affordable settings, we have tested various settings, e.g. mesh topology, resolution and domain size. Mesh convergence tests were conducted for the pitching airfoil (Eq. 3) as baseline simulations. $k_{\text {red }}=0.1$ was used for the 
mesh convergence tests. The initial angle of attack was set to $10^{\circ} \downarrow$. Note that symbols ' $\uparrow$ ' and ' $\downarrow$ ' indicate pitch-up and pitch-down motion respectively, and the pitching moment of pitch-up motion has a negative sign while the pitch-up motion has a positive sign (Eq. 3).

Two types of mesh topology were used for the pitching airfoil cases. The C-type mesh was adopted for the laminar inflow (Fig.1(b)) and the modified multi-block mesh (Fig. 1(c)) was used for the turbulent inflow. The meshes around the both airfoils were C-type, and they were identical within $1.6 \mathrm{c}$ distance from the leading edge. The latter is more convenient for the inflow turbulence generation. The quarter chord point (i.e. the moment centre) was placed at $x=0.25 c$ where $x, y$ and $z$ are the streamwise, cross-flow and spanwise coordinates respectively. The distance of the first grid point to the airfoil surface was $1 \times 10^{-4} c$ near the leading edge and $3 \times 10^{-4} c$ near the trailing edge. Ideally the $y_{1}^{+}<1$ condition should be satisfied for high fidelity simulations. Indeed this condition was fulfilled for most of the pitching time for $k_{\text {red }}=0.1$ and the maximum $y_{1}^{+}$is less than 5 while near the LEV initiation. For the static airfoil simulations (e.g. at $\alpha=10^{\circ}$ ), the maximum $y_{1}^{+}$is less than 1.5 near the leading edge, and $y_{1}^{+}$is less than 0.5 on the most of the surface. Nevertheless, the relatively large $y_{1}^{+}$, albeit only for a short time and a very small region, may cause noticeable influences on LEV developments, and it may be one of the reasons for the deviations in Figs. $4-5$ with $k_{\text {red }}=0.1$. The aspect ratios $\left(\Delta_{x} / \Delta_{y}\right)$ were 15 at the leading edge and 2.3 at the trailing edge. The domain size and number of grid points are summarized in Table 3.

Again, the integrated aerodynamic forces, i.e. lift, drag and moment are 
the focus of this study. Various span widths of the computational domain were tested to check any possible effect. We found that the integrated forces did not show noticeable discrepancy for span widths $0.5 \mathrm{c}$ and $1 \mathrm{c}$, where $\mathrm{c}$ is the chord length; and the span width of the domain was set $0.5 \mathrm{c}$ for most of the computation. Symmetric boundary conditions were applied on the two lateral boundaries. Arguably the simulations of a pitching airfoil are less sensitive than those of a static airfoil. Other boundary conditions are tabulated in Table 2. It is to be noted that in [25] a domain width $0.2 \mathrm{c}$ was used for a static NACA0012 airfoil at $R e_{c}=5 \times 10^{4}$.

Fig. 2 shows the lift and moment hysteresis. Data in Fig. 2 are taken after $\alpha$ has reached $0^{\circ} \uparrow$ for the first time, which corresponds to $t U_{\infty} / c=12$. Only the first cycle after the first $\alpha=0^{\circ} \uparrow$ for all cases is shown in Fig. 2, because we found that the hysteresis from successive cycles matched well in general with that of the first cycle. It is to be noted that the data of the successive cycles of PC5 are in even better agreement with those of the other cases, e.g. PC1 and PC2.

Fig. 3 shows that a strong free shear layer is developed near the leading edge. It is crucial that the mesh is fine enough to capture the free shear layer. Thus the effect of the resolution in the cross-flow (i.e. PC1 and PC2) and chordwise (i.e. PC1 and PC3) directions were tested. The effect of the resolution in the spanwise direction (PC1, PC4 and PC5) was also investigated. Cases PC3 and PC6 were set to investigate the domain width effect on the hysteresis.

There is a noticeable deviation for case PC5 during the downstroke ( $\alpha \sim$ $20^{\circ} \downarrow$ ) in Fig. 2. We have looked into the successive cycles (not shown 
here) carefully. We noticed that the magnitude of the deviation of the lift coefficient was similar to that of the hysteresis fluctuations at $\alpha \sim 20^{\circ} \downarrow$, while the deviation of the first cycle (i.e. in Fig. 2) was the largest. This was because the air flow during the downstroke was more unsteady than during the upstroke. Given these uncertainties, the results of all cases agree reasonably well with each other. The angle where the maximum lift occurs is around $23^{\circ} \uparrow$ and the hysteresis loop has an almost identical shape for all cases. Thus it is a compromise in terms of accuracy and efficiency to choose the mesh for case PC5 as the baseline mesh in the following sections.

It is to be noted that we have performed a mesh convergence test rigorously for a static NACA0012 airfoil with the same Reynolds number (e.g. in Sec. 6.3 in [26], and [27]). Based on the static airfoil data, the resolution along the chordwise and wall-normal directions for case PC5 was nearly the same as that for the static case. The resolution in the spanwise direction was coarser for case PC5, and the grid aspect ratio $\Delta z / \Delta y$ is much greater than the limit set for LES of turbulent flows over a stationary wall. However, our mesh convergence tests for the pitching airfoil showed that the LES was not sensitive to the resolution in the spanwise direction as far as the lift, drag and moment coefficients were of major concerns. This is because the leading edge vortex, which is highly correlated in the spanwise direction, has a dominant influence on dynamics of a pitching airfoil compared to the small turbulence motions in the vicinity of the wall. 


\section{Dynamic stall events}

At a certain pitching angle which exceeds the static stall point, the flow on the airfoil is still attached, which is referred as "stall delay". As the pitching angle continues to increase, the lift and moment change rapidly as the flow starts to detach, which is the so-called "dynamic stall". Complex flow phenomena are investigated by analysing surface pressure, skin friction, pitching moments and flow fields.

Based on the mesh used for case PC5, the effect of the reduced frequency on the forces and moments hysteresis is investigated. From $t U_{\infty} / c=12$ $\left(\alpha=0^{\circ}\right)$, phase-averaging was performed over three cycles. Figs. 4 and 5 show phase-averaged $C_{L}, C_{D}, C_{M}$ at $k_{r e d}=0.025,0.05$ and 0.1 . Note that the experimental data [7] were obtained through averaging over 100 cycles. Though the three-cycles phase-averaged LES data in Figs. 4 and 5 were not fully converged, the stall angle and size of hysteresis were found nearly the same at each cycle. Therefore, a longer phase average would not be expected to improve the agreement between the LES and experimental data. The load hysteresis from the simulations are integrated over the airfoil surface, while those from the experiment were integrated over a number of pressure taps along a streamwise line on the airfoil surface. It is also to be noted that adopting a domain width $1 c$ (PC6) did not present noticeable differences in force and moment hysteresis compared to using a domain width $0.5 c$ (PC3). This again confirms the same conclusion in $[28,25]$.

In the experiment [7], $C_{L}, C_{D}$ and $C_{M}$ were calculated from pressure tap measurements and these taps were placed at $0<x / c<0.8$ over the airfoil surface. For a rigorous comparison, two sets of calculations were used 
to obtain the surface forces. The first set integrated over the entire airfoil surface $(0 \leq x / c \leq 1)$ (LES1), while the second integrated over a part of the airfoil surface (i.e. $0 \leq x / c \leq 0.8)(\operatorname{LES} 2)$ which was the same as that of the experiment. The case LES2 shows a slightly better agreement with the reference data than the case LES1 (Figs. 4 and 5), in particular for the moment coefficient $C_{M}$. Maximum and minimum aerodynamic coefficients are summarized in Table 4 and compared with the experimental data.

The discrepancy between the LES data and the wind tunnel measurements [7] was not small for $k_{r e d}=0.1$ (Figs. 4 (c,f) and 5 (c)). Specifically, the maximum lift coefficient $C_{L, \max }$ was under-predicted by 0.33 , which was associated with the first LEV generation and its convection. The subsequent differences during the downstroke (hysteresis loop and second LEV) seemed to be consequences of the deviation in the first LEV predictions. It was noted that the deviation between the calculations and experiments tended to decrease as $k_{\text {red }}$ decreased. Fig. 4 (a,b,d,e) show a better agreement in $C_{L}$ and $C_{D}$

In the wind tunnel experiments at a high reduced frequency, it would be very challenging to measure the instantaneous surface pressure during the downstroke due to the massive LEV induced separated flow. In contrast, LES should not suffer from a technical limit to calculate the surface pressure as long as the large flow structures are resolved accurately. To verify this, mesh convergence tests were conducted and showed that a greater domain width (i.e. PC6 in Table 3) did not show noticeable discrepancy on the hysteresis loop. Note that four times finer mesh (i.e. PC4) in the spanwise direction showed almost same hysteresis with case PC5. Though the mesh 
convergence tests were conducted, we emphasize again that it is difficult to capture all of the details of such a complicated behaviour of the LEV. Thus far we cannot speculate any other sources in LES which might be responsible for the discrepancy at high reduced frequencies. In this study, it is unlikely that we are able to entirely resolve this uncertain point arising from the comparison between the experiments and LES. Therefore, we focus on the reduced frequency $k_{\text {red }}=0.05$ for the rest of this paper.

It would be very valuable to see error bars for the experimental data. Unfortunately, error data are not available in [7]. Overall, all aerodynamic coefficients agree well with the measurements. As the reduced frequency increases, the magnitudes of the peaks of $C_{L}, C_{D}$ and $C_{M}$ increase and the angle of the maximum lift increases. The same trend was also reported in the literature $[2,3]$.

\subsection{Laminar separation bubble diminishing and boundary layer suppression}

Fig. 6 shows a comparison of pressure coefficient $C_{p}$ between the static and pitching airfoils at a similar angle of incidence. For the pitching airfoil at $k_{\text {red }}=0.05$, the pressure coefficient and vorticity field do not show any indication of laminar separation bubbles at the leading edge. In contrast, Fig. 6 shows a negative plateau of the measured $C_{p}$ of a static airfoil which is due to a laminar separation bubble observed in the experiments [7]. The instantaneous spanwise component of vorticity at the middle cross-section confirms that the boundary layer is attached on the pitching airfoil at this angle of incidence. These firmly confirm that during the pitch-up process, the boundary layer on the suction side of the airfoil is suppressed and the size of the laminar separation bubbles significantly diminishes or completely 
disappears.

Note that static stall occurs at $\alpha \approx 13^{\circ}$ [7] at the given conditions. Fig. 7 shows the contours of instantaneous velocity magnitude for the static and pitching airfoils at $\alpha \approx 10^{\circ}$. The boundary layer thickness on the pitching airfoil is significantly thinner than that on the static airfoil near the trailing edge. Thus the boundary layer on the pitching airfoil is suppressed compared with that on the static airfoil at the same angle of incidence.

This boundary layer suppression is mainly due to the time lag of the boundary layer development on moving objects [29, 15]. When the airfoil is pitching, the flow around it at a given geometric angle of attack (angle between the freestream velocity direction and chord line) does not 'see' the same flow topology as that around the static airfoil at the same geometric angle of attack. This is because the flow over the pitching airfoil 'remembers' its history. During the upstroke, the boundary layer of the pitching airfoil seems to be suppressed because it 'remembers' the early flow topology which is produced at smaller angles of incidence.

The pitching airfoil passes the static stall angle $\alpha \approx 13^{\circ} \uparrow[7]$ without any discernible change in the lift coefficient slope for all reduced frequency ranges as shown in Fig. 4. This is stall delay, which is due to a combination of the aforementioned laminar separation bubble diminishing and the lag of the boundary layer development (i.e. boundary layer suppression).

\subsection{Leading edge vortex}

As angle of attack increases, the first leading edge vortex (LEV) is initiated. When LEV is generated and convects downstream, a lift increase follows. The reason for this increment is because the LEV greatly enlarges 
the effective camber of the airfoil [30]. The LEV initiation, convection and its influence to the lift, drag and pitching moment are the most important mechanism in dynamic stall. However, these are not well understood yet.

The characteristics of the first LEV are quantified for $k_{\text {red }}=0.025-0.1$. The convection speed of the leading edge vortex $\left(U_{L E V}\right)$ with respect to the chord line, can be quantified by measuring the travelling time and the corresponding distance between the pressure peaks on the suction side of the airfoil [31]. Fig. 8 shows the pressure and skin-friction coefficients at two different angles of attack. A strong leading edge vortex presents peaks of $C_{p}$ and $C_{f}$ which are marked in the figure. Then $U_{L E V}$ is estimated by using the time interval between the two incidences and the distance between the two peak points. The negative peaks on the pressure contours at the same incidence in Fig. 9 (dashed circles) confirm the correlation between the LEV and the surface forces. By using this estimation, it is shown that $U_{L E V} \approx 0.25 U_{\infty}$ for $k_{\text {red }}=0.025-0.1$. It is to be noted that the LEV convection speed is independent of $k_{\text {red }}$ in that range. Green et al. (1992) [31] measured the LEV convection speed with various types of airfoils. They concluded that the LEV convection speed was independent of the airfoil motion, and also reported that $U_{L E V} \approx 0.26 U_{\infty}-0.31 U_{\infty}$ at the maximum pitch angle $\alpha_{\max } \approx 25^{\circ}$ for the NACA 0012 airfoil. A similar LEV convection velocity $U_{L E V} \approx 0.3 U_{\infty}$ was also reported by another group [32]. Considering the uncertainties in determining the vortex cores, the difference in the LEV convection speed between the current case and those in the literature is relatively small.

Fig. 10 shows $C_{L}$ versus phase angle at $k_{\text {red }}=0.025,0.05$ and 0.1 . The 
large dots represent the peak lift due to the leading edge vortex generating and convecting over the upper airfoil surface. The magnitudes of the first and second peaks decrease as the reduced frequency decreases. The maximumlift angle decreases towards the static stall angle as the reduced frequency decreases. At a very low pitching frequency, a quasi-steady state would be expected and the lift coefficients for the pitching airfoil would be the same as those for the static case at the same angle of incidence. McAlister et al. (1978) [33] reported that the aerodynamic forces are quasi-steady for $k_{\text {red }}<0.004$.

The shedding frequency between the first and second leading edge vortices are characterized by the Strouhal number,

$$
S t=\frac{f_{s} c \sin \alpha_{L E V}}{U_{\infty}},
$$

where $f_{s}$ is the shedding frequency and $\alpha_{L E V}$ is the mean angle of attack between the first and second LEV peaks. The Strouhal number for the present study $\left(k_{\text {red }}=0.025-0.1\right)$ is approximately 0.1 , which is much lower than the well-known bluff-body shedding frequency, i.e $S t \approx 0.2$ [34]. Zaman et al. (1989) [35] reported that the Strouhal number of the flow over a static airfoil varies depending on angle of attack, e.g. $S t \approx 0.2$ when $\alpha>18^{\circ}$ (post-stall) and $S t \approx 0.02$ when $\alpha<15^{\circ}$ (pre-stall). The shedding frequency for the pitching airfoil lies between those of the pre- and post-stall regimes. This may well explain that the shedding frequency shows a combined characteristics of both regimes, because the pitching angles vary across both pre- and post-stall regimes. 
To understand important features of dynamic stall, Figs. 11 and 12 show snapshots at typical phase angles and reduced frequency $k_{\text {red }}=0.05$ of contours of the spanwise component of vorticity and pressure field respectively. Each snapshot is given an identification number (ID) in Figs. 11 and 12. The IDs are marked on the lift and moment coefficients profiles in Fig. 13. These snapshots are summarized below:

1. $\alpha=10^{\circ} \uparrow$, the laminar separation bubble and boundary layer are suppressed compared to those on a static airfoil at the same angle of attack;

2. $\alpha=13^{\circ} \uparrow$, the lift continues increasing linearly after exceeding the static stall angle without a discernible change of the lift coefficient slope;

3. $\alpha=18.2^{\circ} \uparrow$, the moment coefficient starts to drop rapidly, i.e. moment stall, whereas the lift coefficient slope increases rapidly and low pressure is formed at the suction side as the first leading-edge vortex is initiated;

4. $\alpha=19.9^{\circ} \uparrow$, the lift coefficient reaches the global maximum and starts to decrease whereas the moment coefficient reaches the global minimum, and a large area of low pressure at the suction side is observed while the first leading-edge vortex convecting downstream;

5. $\alpha=22.4^{\circ} \uparrow$, the lift coefficient increases again (after having passed the first local minimum) as the second leading-edge vortex (LEV) is generated and convected downstream, while the first LEV has detached and passed the trailing edge;

6. $\alpha=23.3^{\circ} \uparrow$, the lift coefficient passes the second local maximum as the second leading-edge vortex passes over the first half chord, while 
the moment coefficient reaches its second local minimum (an evident tip vortex is formed which is entrained by the leading-edge vortex as it passes over the trailing edge);

7. $\alpha=24.8^{\circ} \uparrow$, a small increase of the lift coefficient is observed due to the generation and convection of the third LEV;

8. $\alpha=25^{\circ}$, the maximum angle of attack is reached and a large vortex is shed;

9. $\alpha=10.2^{\circ} \downarrow$, the flow begins to be attached, in particular at the first half chord;

10. $\alpha=4^{\circ} \downarrow$, the flow is fully attached.

Spanwise vorticity component and instantaneous streamlines show great similarities with the experimental data of Raffel et al. (1995) [36] in Fig. 14. The spanwise vorticity component in the current simulation reveals some fluctuation distributions within the leading edge vortex, which were also observed in the experiment (see Figs. 14(a) and 14(c)). Such details were not found in the RANS calculations by Wang et al. (2010) [13]. This is not surprising because RANS is not designed to model genuine unsteady flows. This demonstrates the capability of the LES techniques.

For a comparison of using various approaches describing the LEV, instantaneous iso-contours of a vortex identifier, i.e. $\lambda_{2}$ criterion are presented in Fig. 15. $\lambda_{2}$ denotes the second eigenvalue of the matrix $S_{i k} S_{k j}+\Omega_{i k} \Omega_{k j}$, where $S_{i j}=0.5\left(\partial u_{i} / \partial x_{j}+\partial u_{j} / \partial x_{i}\right)$, and $\Omega_{i j}=0.5\left(\partial u_{i} / \partial x_{j}-\partial u_{j} / \partial x_{i}\right)$. One snapshot for case PC5 at $k_{\text {red }}=0.1, \alpha=23.3^{\circ} \uparrow$ is plotted in Fig. 15. As the pitching angle increases, a large vortex is generated from the leading edge, evolves and convects downstream along the upper surface, and is finally de- 
tached from the trailing edge. The size of the vortex is comparable with the chord length. Overall, these are consistent with the other approaches describing the LEV, such as Fig. 14 (a,b).

\section{The effect of freestream turbulence}

\subsection{A brief of the divergence-free inflow turbulence generation}

The approach [37], which is denoted XC, imposes correlations using an exponential function to satisfy the prescribed space and time integral length scales. It is a synthetic turbulence generation method. The inlet velocities can be written as,

$$
u_{i}=U_{i}+a_{i j} u_{*, j},
$$

where $i, j=1,2,3 . u_{i}$ is an instantaneous velocity which is imposed at the inlet boundary, $U_{i}$ is a prescribed mean velocity, $a_{i j}$ is a prescribed tensor (Eq.13) and $u_{*, j}$ is an auto-correlated fluctuation satisfying the prescribed integral length scales, but with a zero mean, zero cross-correlations and a unit variance. Lund et al. [38] suggested a form for $a_{i j}$, using Cholesky decomposition of the prescribed Reynolds stress tensor, $R_{i j}$,

$$
a_{i j}=\left(\begin{array}{ccc}
\sqrt{R_{11}} & 0 & 0 \\
R_{21} / a_{11} & \sqrt{R_{22}-a_{21}^{2}} & 0 \\
R_{31} / a_{11} & \left(R_{32}-a_{21} a_{31}\right) / a_{22} & \sqrt{R_{33}-a_{31}^{2}-a_{32}^{2}}
\end{array}\right) .
$$

This matrix builds scaling and cross-correlations based on $u_{*, j}$ in Eq. 12 . To impose correlations on random sequences, the $\mathrm{XC}$ approach adopted an 

correlations

$$
\psi_{m}=\sum_{j=-N}^{N} b_{j} r_{m+j}
$$

474

exponential function instead of a Gaussian function used in the early digitalfilter based methods. The digital filter method was used to generate spatial where $N=2 n, n=I / \Delta x, \Delta x$ is grid size and $I$ is integral length scale. $\psi_{m}$ is the intermediate velocity field and $r_{j}$ is a one-dimensional random number sequence with a zero mean and a unit variance. $\psi_{m}$ is a one-dimensional number sequence with a zero mean, a unit variance and spatial correlations. Note that the subscripts, $m, j$, are the position indices. The constant $b_{j}$ is estimated as,

$$
b_{j}=\frac{b_{j}^{\prime}}{\left(\sum_{l=-N}^{N}{b^{\prime}}_{l}^{2}\right)^{1 / 2}} \text { with } b_{j}^{\prime}=\exp \left(-\frac{\pi|j|}{2 n}\right) .
$$

It is straightforward to generate spatial correlations for a two dimensional space (cf. Eq.14) as,

$$
\psi_{m, l}=\sum_{j=-N}^{N} \sum_{k=-N}^{N} b_{j} b_{k} r_{m+j, l+k} .
$$

It is to be noted that only one slice of two dimensional data, $\psi_{m, l}$, is generated at each time step. Based on these data, a time correlation is built using the efficient forward stepwise relation,

$$
u_{*, i}(t+\Delta t)=u_{*, i}(t) \exp \left(-\frac{C_{X C} \Delta t}{T}\right)+\psi_{i}(t)\left[1-\exp \left(-\frac{2 C_{X C} \Delta t}{T}\right)\right]^{0.5},
$$


where the constant $C_{X C}=\pi / 4$ and $T$ is the Lagrangian time scale which is estimated using $T=I / U_{1}$ where, again, $I$ is a turbulence integral length scale and $U_{1}$ is a mean convective velocity. Note that in Eq.17 the subscript $i$ is a vector index, i.e. $i=1,2,3$. The $\mathrm{XC}$ method generates synthetic turbulence by using Eqs. 12 - 17. By using exponential correlations, in particular in the streamwise direction, it significantly reduces the computational time compared to the early digital filter based approaches. The XC method is a combination of the digital filter method and the forward stepwise methods and is also denoted Hybrid Forward Stepwise (HFS) approach.

Based on the XC method, Kim et al.(2013) [39] develop a divergencefree approach - denoted XCDF thereafter. After the predictor step in the PISO solver for unsteady flows, synthetic turbulence fluctuations are inserted into the source term of the Poisson equation in one of the corrector steps. Hence the divergence-free condition was achieved without solving an additional Poisson equation. The XCDF approach significantly improve the prediction of surface pressure fluctuations. More details of the implementation of the XCDF approach is given in the following sub-section.

\subsection{Upstream turbulence}

To characterize upstream turbulence, a new mesh was generated in which the upstream region of the domain was the same as the modified mesh as shown in Fig. 1(c). With the airfoil removed, this is denoted an 'empty box case'. The downstream half of the mesh of the 'empty box case' was the same as the upstream half. The boundary conditions, numerical schemes and domain size were the same as those for case PC5.

The PC5 mesh (Table 3) was adopted for $k_{\text {red }}=0.05$, and the divergence- 
free synthetic turbulence inflow approach XCDF was applied on a 2-D transverse plane placed at $x / c=-7$, or at $7 \mathrm{c}$ upstream from the leading edge of the airfoil in case PC5 (i.e. the airfoil included in the domain).

Two different turbulence intensities, $T I_{0}=5 \%$ and $10 \%$, were used where suffix ' 0 ' denotes the input variable. Then the same turbulence characteristics were used for the flow over the pitching airfoil. The turbulence length scales in the atmospheric boundary layer ranges from $0.001 \mathrm{~m}$ to $500 \mathrm{~m}$ [40]. The turbulence scales which are greater than the chord length can be considered as large-scale unsteadiness [41]. The integral length scales were set to be comparable with the chord length. The integral length scales for the XCDF model were $I_{i 1}=0.3 c, I_{i 2}=0.15 c$ and $I_{i 3}=0.15 c$ in the streamwise, cross-flow and spanwise directions, respectively, where $i$ indicates the velocity components. The integral length scales $I_{i j}$ are defined as below,

$$
I_{i j}=\int_{0}^{r_{i j, 0.1}} C_{i}\left(r \hat{e}_{j}\right) d r,
$$

where $C_{i}\left(r \hat{e}_{j}\right)$ is the correlation function. $i$ and $j$ correspond to the components of the velocity vector and directions respectively, and $r_{i j, 0.1}$ is the separation distance for $C_{i}\left(r \hat{e}_{j}\right)=0.1$.

The grid size normalized by the integral length scale was $\Delta x=0.333 I_{11}$, $\Delta y=0.252 I_{11}$ and $\Delta z=0.083 I_{11}$. The time step, normalized by $I_{11}$ and $U_{\infty}$, was $\Delta t \times U_{\infty} / I_{11}=0.0133$. For easy reading, the coordinates for the empty box case were normalized by $I_{11}$. The synthetic turbulence was imposed at $x / I_{i i}=-23.3$. The leading edge of the pitching airfoil would be later placed at $x / I_{i i}=0$. The turbulent characteristics at $x / I_{11}=-23.3$ and $x / I_{11}=0$ are summarized in Table. 5. 
Fig. 16 shows one-dimensional compensated energy spectra of the streamwise velocity fluctuations normalized by the local turbulent kinetic energies at $x / I_{11}=0$. The inertial subrange (i.e. a plateau) is visible for the two cases. The highest wavenumber that can be resolved by the current resolution is $\kappa_{\max } I_{11}=\frac{1}{2} \frac{2 \pi}{\Delta x} I_{11} \approx 9.4$ but $E_{11}$ starts to drop $\kappa I_{11} \approx 2.5$. This is associated with the SGS model, mesh resolution, filtering method and numerical schemes. Further careful study has been performed and it was found that the grid size was about two orders of magnitude greater than the Kolmogorov dissipation scale.

It is also to be noted that the mesh in the region where the airfoil is placed will be refined in Section 5.3. This will improve the simulation of the turbulence decay.

The ratios of turbulence fluctuations are $\frac{u_{r m s}^{\prime}}{v_{r m s}^{\prime}} \approx 2$ and $\frac{u_{r m s}^{\prime}}{w_{r m s}^{\prime}} \approx 3$ at $x / I_{11}=$ 0 where the airfoil will be placed. Again the purpose of this study is to investigate the effect of the given freestream turbulence characteristics on the flow over a pitching airfoil rather than to predict an accurate decay of homogeneous isotropic turbulence. Therefore turbulence intensities $5 \%$ and $10 \%$ with current configurations were used to investigate the pitching airfoil flows. The turbulence characteristics at $x / I_{11}=0$ are considered as the effective freestream turbulence.

\subsection{The effect of turbulence intensities}

It was observed that the inflow turbulence significantly suppressed the separation bubble of flows around a static airfoil, which was certainly because the greater momentum of turbulent flows delayed the occurrence of the inverse pressure gradient over the suction side. It is of great interest to 
investigate the effect of the free-stream turbulence on flows around a pitching airfoil. Again, the synthetic turbulence was imposed on the transverse plane at $x / c=-7$ in the upstream region of the pitching airfoil for $k_{\text {red }}=0.05$. Fig. 17 shows the effect of freestream turbulence on aerodynamic characteristics. In general, the freestream turbulence does not significantly change the force and moment hysteresis at the given conditions. The angles for the maximum lift, drag and minimum moment are nearly the same as those for the smooth flow case $T I_{0}=0 \%$. The magnitudes of maximum drag and minimum moment slightly decrease with the increase of the inflow turbulence intensity.

The drag coefficients in the pre-stall regime show no discernable difference between the laminar and turbulent inflow cases. This is interesting since usually turbulence enhances the skin friction. Fig. 17(b) shows that the drag coefficient increases rapidly at a large angle of attack, e.g. $\alpha=10^{\circ}$. At such large angles, the drag is mainly contributed by the pressure difference (i.e. form drag), and therefore the variation of the contribution of the skinfriction is hard to discern in Fig. 17(b).

The most evident impact of freestream turbulence on the lift coefficient occurs during the downstroke (Fig. 17(a)), when the lift coefficient increases evidently as the inflow turbulence intensity increases. The average increment for $T I_{0}=10 \%$ is $\Delta C_{L} \approx 0.2$ during the downstroke. Similar experimental results were reported by Amandolèse and Széchényi (2004) [1], who measured the effects of upstream turbulence on the flow over a pitching airfoil, and found that the maximum lift angle showed little change while a clear lift increment was observed during the downstroke as the inflow turbulence 
intensity increased. In the current study, the lift increment at most of the phase angles during the downstroke is evident. Nevertheless, the difference at some phase angles during the downstroke is within the range of uncertainties, in particular for the cases $T I_{0}=5^{\circ}$ and $T I_{0}=10^{\circ}$. Another evident effect of the inflow turbulence is that the re-attachment of the flow occurs much earlier (e.g. approximately $4^{\circ}$ earlier for the case $T I_{0}=10 \%$ ) than that for the smooth inflow case during the downstroke.

Fig. 18 shows typical snapshots of the instantaneous spanwise component of vorticity at the mid-span cross-section with laminar (i.e. $T I_{0}=0$ ) and turbulent (i.e. $T I_{0}=10 \%$ ) inflow conditions. For the laminar inflow case (Fig. $18 \mathrm{a}-\mathrm{d}$ ), the flows are fully detached and Kelvin-Helmholtz vortex is observed along with the free shear layer starting from the leading edge. For the turbulent inflow (Fig. 18 e-h), the turbulence convected from upstream, in which the length scales are comparable with the chord length, breaks down the separation bubble (e.g. Fig. 18(f)). Thus the size of the separated region tends to decrease and the re-attachment occurs earlier. This leads to an increase of the lift during the downstroke. In particular, the influence of freestream turbulence is evident near the leading edge at $\alpha=14.2^{\circ} \downarrow$ (Fig. 18(h)) compared to the smooth inflow at the same angle of attack (Fig. 18 d).

Time series of the instantaneous streamwise velocity at $x / c=0.75$ and $y / c=0.2$ at the central plane were sampled during one period, and are shown in Fig. 19(a). In general, the signals from both the laminar and turbulent inflow cases show a high correlation. For the laminar inflow case during $\omega t<0^{\circ}$ or $\omega t>180^{\circ}$, the velocity signal is smooth with its magnitude close 
to that of the freestream velocity. In contrast for the turbulent inflow case, velocity fluctuations are evident during the same phase angle ranges. During $0^{\circ}<\omega t<180^{\circ}$, the velocity deficit due to the boundary layer separation and leading edge vortex shedding is evidently less for the turbulent inflow case compared to that for the laminar inflow case. This is because the upstream turbulence disturbs the leading edge vortex and enhances the mixing between the freestream flow and the local boundary layer flows.

Fig. 19(b) shows the energy spectra using the same streamwise velocity as shown in Fig. 19(a). The first peak corresponds to the pitching frequency $\kappa c=0.1$. The 2 nd peak corresponds to the LEV shedding mode. The magnitudes of the first and second peaks for the laminar inflow case are approximately twice of those respectively for the turbulent inflow case. This is because there are much more high frequency fluctuations for the latter as shown in Fig. 19(a). Fig. 19(b) shows that an inertial sub-range - a $-5 / 3$ slope is evident for the both cases. There is a clear spectral gap between the pitching mode and inertial sub-range for the laminar inflow case but it is less distinctive for the turbulent inflow case. The latter may raise challenges for Unsteady RANS approaches, in particular when the pitching frequency increases.

To our best knowledge, this was the first attempt at applying LES to investigate the effect of freestream turbulence on dynamic stall at the moderate Reynolds number, i.e. $R e=135,000$. The flows around a pitching aerofoil consist of small scale turbulence motions and large scale motions. The latter has the same time scales as the unsteady external forcing. LES for such flows is denoted 'unsteady LES', whereas LES for turbulent flows subjected to a 
steady external forcing, e.g. flows around a stationary aerofoil, is denoted 'steady LES'. In this study, a few cycles of the pitching motion are required to simulate using the 'unsteady' LES, which is much more expensive than the 'steady' LES studies.

\section{Conclusion}

To understand the effect of the upstream large turbulence eddies (e.g. greater than the diameter of the wind turbine disk) on the wind turbine aerodynamics, the dynamic stall events on a pitching airfoil have been investigated using high fidelity numerical simulations. The lift, drag and moment hysteresis show good agreement with experimental data [7] at three different reduced frequencies, $k_{\text {red }}=0.025,0.05$ and 0.1 , but better at lower $k_{\text {red }}$. The laminar separation bubble diminishing and boundary layer suppression on the pitching airfoil are illustrated through investigating the surface pressure, skin friction and flow visualisation. The generation and motion of the leading edge vortex are quantified in terms of its shedding frequency and convection speed, and are compared with those in literature.

To examine the impact of upstream small-scale turbulence (i.e. in the order of the chord length of the wind turbine blade) on the wind turbine aerodynamics, freestream synthetic turbulence was implemented on a 2-D transverse plane at $x / c=-7$ upstream of the leading edge of static [28] and pitching airfoils, and the results were compared with those for the laminar inflow case. For the static airfoil, the separation bubble is diminished as the turbulence level increases resulting in an increase of the lift to drag ratio.

The effect on the aerodynamic forces of the pitching airfoil was the focus 
in this paper. For the pitching airfoil, the magnitudes of the maximum drag and minimum moment decrease with the increase of the freestream turbulence. This is mainly attributed to the suppression of separated flows in turbulent flows. The most evident impact of freestream turbulence on the lift coefficient occurs during the downstroke, i.e. an approximate $50 \%$ increase for a turbulence intensity $6 \%$ immediately upstream of the airfoil compared to the smooth inflow flow. The snapshots of the vorticity fields at different incidence also confirm that the freestream turbulence has a nonnegligible impact on the flow around the pitching airfoil.

The power spectral density of the streamwise velocity component sampled at one point in the wake, presents the peak mode corresponding to the pitching frequency for both the laminar and turbulent inflow cases. For the latter, the energy separation between the pitching mode and the inertial sub-range is less apparent compared to the former. This would be even worse with a higher pitching frequency. Thus it is extremely challenging for using unsteady RANS to model the latter, in particular at a high pitching frequency. It is concluded that the LES approach is desirable when the flow is highly separated and subjected to upstream turbulence.

To the authors knowledge, this is the first attempt for applying an LES calculation on the flow over a pitching airfoil at the moderate Reynolds number, i.e. $\operatorname{Re}=135,000$, considering the freestream turbulence effects. Requiring massive computational resources for such work makes these tasks even more challenging. Further work on high reduced frequencies should be conducted in the future to rule out any uncertainties in this aspect. In summary, the capability of Large-Eddy Simulation is successfully demonstrated 
for highly separated flows at deep stall.

\section{Acknowledgements}

The authors are grateful to Prof Ian Castro for his advice throughout the project and comments on the manuscript of this paper. The authors are grateful to Prof Timothy Lee for his advice and valuable comments during the project, and Prof Kenichi Rinoie for sharing the raw data. YK acknowledges the provision of a Ph.D studentship from the Faculty of Engineering and the Environment, University of Southampton. The authors also thank to the two anonymous reviewers for their comments. The computations were performed on IRIDIS3 at the University of Southampton and the UK National Supercomputing Service HECToR supported by the EPSRC RAP.

\section{References}

[1] Amandolèse X, Széchényl E . Experimental study of the effect of turbulence on a section model blade oscillating in stall. Wind Energy $2004 ; 7: 267-82$.

[2] Carr LW, McAlister W, McCroskey WJ . Analysis of the development of dynamic stall based on oscillating airfoil experiments. Tech. Rep. TN D-8382; NASA; 1977.

[3] McCroskey WJ . The phenomenon of dynamic stall. Tech. Rep. TR 81-A-6; NASA; 1981.

[4] McAlister KW, Carr LW . Water-tunnel visualizations of dynamic stall. J Fluids Eng 1979;101:376-80. 
[5] McCroskey WJ, McAlister KW, Carr LW, Pucci SL . An experimental study of dynamic stall on advanced airfoil sections. Volume 1: Summary of the experiment. Tech. Rep. TR-82-A-8; NASA; 1982.

[6] Butterfield CP, Hansen AC, Simms D, Scott G . Dynamic stall on wind turbine blades. Tech. Rep. TP-257-4510; NREL; 1991.

[7] Lee T, Gerontakos P . Investigation of flow over an oscillating airfoil. J Fluid Mech 2004;512:313-41.

[8] Piziali RA . 2-D and 3-D oscillating wing aerodynamics for a range of angles of attack including stall. Tech. Rep. TR 94-A001; NASA; 1994.

[9] Ramsay RR, Hoffmann MJ, Gregorek GM . Effects of grit roughness and pitch oscillations on the S809 airfoil. Tech. Rep. TP-442-7817; NREL; 1995.

[10] Shipley DE, Miller MS, Robinson MC . Dynamic stall occurrence on a horizontal axis wind turbine blade. Tech. Rep. TP-442-6912; NREL; 1995.

[11] Ekaterinaris JA, Menter FR . Computation of oscillating airfoil flows with one- and two-equation turbulence models. AIAA J 1994;32(12):2359-65.

[12] Barakos GN, Drikakis D . Unsteady separated flows over maneuvering lifting surfaces. Phil Trans R Soc Lond A 2000;358:3279-91. 
[13] Wang S, Ingham DB, Ma L, Pourkashanian M, Tao Z . Numerical investigations on dynamic stall of low Reynolds number flow around oscillating airfoils. Comput Fluids 2010;39:1529-41.

[14] Ekaterinaris JA, Platzer MF . Computational prediction of airfoil dynamic stall. Prog Aerospace Sci 1997;33:759-846.

[15] Leishman JG . Principles of helicopter aerodynamics; chap. 9. Cambridge University Press; 2000, p. 378-417.

[16] Nagarajan S, Hahn S, and Lele S . Prediction of =sound generated by a pitching airfoil: A comparison of rans and les. AIAA Paper 20006;20062516.

[17] Haase W, Braza M, Revell A . Desider - a european effort on hybrid rans-les modelling. Notes on Numerical Fluid Mechanics and Multidisciplinary Design 103 2009;103.

[18] Rinoie K, Takemura N . Oscillating behaviour of laminar separation bubble formed on an aerofoil near stall. The Aeronaut J 2004;108:15363.

[19] Lissaman PBS . Low-Reynolds-number airfoils. Ann Rev Fluid Mech $1983 ; 15: 223-39$.

[20] Inagaki M, Kondoh T, Nagano Y . A Mixed-Time-Scale SGS model with fixed model-parameters for practical LES. J Fluids Eng 2005;127:1-13.

[21] Krishnan L, Sandham ND, Steelant J . Shock-wave/boundary-layer interactions in a model scramjet intake. AIAA J 2009;47:1680-91. 
[22] Jasak H . Error analysis and estimation for the finite volume method with application to fluid flows. Ph.D. thesis; Imperial College of Science, Technology and Medicine; 1996.

[23] OpenFOAM . User guide v1.7.1. Tech. Rep.; OpenFOAM@; 2010.

[24] Jasak H, Tuković Ž . Automatic mesh motion for the unstructured finite volume method. Transactions of FAMENA 2004;30:1-18.

[25] Jones LE, Sandberg RD, Sandham ND . Direct numerical simulations of forced and unforced separation bubbles on an airfoil at incidence. J Fluid Mech 2008;602:175-207.

[26] Kim Y . Wind turbine aerodynamics in freestream turbulence. Ph.D. thesis; University of Southampton; 2013.

[27] Kim Y, Castro IP, Xie ZT . Large-eddy simulations for wind turbine blade: dynamic stall and rotational augmentation. Ercoftac Series Direct and Large Eddy Simulation IX, J Frohlich et al, Eds, Springer, DOI 101007/978-3-319-14448-1 2015;20:369-75.

[28] Kim Y, Castro IP, Xie ZT . Numerical study of 3-d effects on dynamic stall of a wind turbine blade. Proceedings of the 13th International Conference on Wind Engineering 2011;ICWE13, the Netherlands.

[29] Larsen JW, Nielsen SRK, Krenk S . Dynamic stall model for wind turbine airfoils. J Fluids Struct 2007;23:959-82.

[30] Dickinson MH, Götz KG . Unsteady aerodynamic performance of model wings at low Reynolds numbers. J Exp Biol 1993;174:45-64. 
[31] Green RB, Galbraith RAM, Niven AJ . Measurements of the dynamic stall vortex convection speed. Aeronaut J 1992;:319-25.

[32] Chandrasekhara M, Carr LW . Flow visualization studies of the Mach number effects on dynamic stall of an oscillating airfoil. J Aircraft 1990;27:516-22.

[33] McAlister KW, Carr LW, McCroskey WJ . Dynamic stall experiments on the NACA 0012 airfoil. Tech. Rep. Technical Paper 1100; NASA; 1978.

[34] Roshko A . On the drag and shedding frequency of two-dimensional bluff bodies. Tech. Rep. TN-3169; NACA; 1954.

[35] Zaman KBMQ, McKinzie DJ, Rumsey CL . A natural low-frequency oscillation of the flow over an airfoil near stalling conditions. J Fluid Mech 1989;202:403-42.

[36] Raffel M, Kompenhans J, Wernert P . Investigation of the unsteady flow velocity field above an airfoil pitching under deep dynamic stall conditions. Exp Fluids 1995;19:103-11.

[37] Xie ZT, Castro IP . Efficient generation of inflow conditions for large eddy simulation of street-scale flow. Flow Turb Comb 2008;81:449-70.

[38] Lund TS, Wu X, Squires KD . Generation of turbulent inflow data for spatially-developing boundary layer simulations. J Comput Phys 1998;140:233-58. 
[39] Kim Y, Castro IP, Xie ZT . Divergence-free turbulence inflow condition for large-eddy simulations with incompressible flow solvers. Comput Fluids 2013;84:56-68.

[40] Kaimal JC, Finnigan JJ . Atmospheric boundary layer flows - their structure and measurement. Oxford University Press; 1994,.

[41] Sicot C, Aubrun S, Loyer S, Devinant P . Unsteady characteristics of the static stall of an airfoil subjected to freestream turbulence level up to 16\%. Exp Fluids 2006;41:641-8.

[42] McAlister KW, Pucci SL, McCroskey WJ, Carr LW . An experimental study of dynamic stall on advanced airfoil sections. Volume 2: Pressure and force data. Tech. Rep. TR-82-A-8; NASA; 1982.

[43] Schreck S, Robinson M . Blade three-dimensional dynamic stall response to wind turbine operating condition. J Sol Energy Eng 2005;127:488-95.

[44] Pope SB . Turbulent flows; chap. 6. Cambridge; 2000, p. 182-263. 
Table 1: Summary of literature on dynamic stall. $\alpha_{0}$ and $\alpha_{1}$ are the mean angle and pitching amplitude. The tip speed ratio (TSR) is $\mathrm{TSR}=r \Omega / U_{\infty}$ and $k_{\text {red }}$ from the rotating blades are based on $U_{\text {eff }}$ in Eq. 2

\begin{tabular}{|c|c|c|c|c|c|c|c|}
\hline & Authors & Method & $\operatorname{Re}\left[10^{6}\right]$ & $k_{\text {red }}$ & Airfoils & $\alpha_{0}\left[{ }^{\circ}\right]$ & $\alpha_{1}\left[^{\circ}\right]$ \\
\hline \multirow{14}{*}{$\begin{array}{l}\text { Oscillating } \\
\text { airfoil }\end{array}$} & Carr et al. [2] & Experiment & $1.3-3.5$ & $0.02-0.25$ & NACA0012 & $6-15$ & $6-14$ \\
\hline & McCroskey et al. [5], McCalis- & Experiment & $0.5-4$ & $0.05-0.25$ & NACA0012, & $6-15$ & $6-14$ \\
\hline & ter et al. $[42]$ & & & & 7 more types & & \\
\hline & Piziali [8] & Experiment & 2 & $0.04-0.2$ & NACA0015 & $4-17$ & $2-5$ \\
\hline & Ekaterinaris and Menter [11] & CFD & $2-4$ & 0.1 & NACA0012, & $4-15$ & $4.2-5$ \\
\hline & & & & & NACA0015 & & \\
\hline & Raffel et al. [36] & Experiment & 0.373 & 0.15 & NACA0012 & 15 & 10 \\
\hline & Ramsay et al. [9] & Experiment & $0.75-1.4$ & $0.025-0.1$ & S809 & $8-20$ & $5.5-10$ \\
\hline & Barakos and Drikakis [12] & $\mathrm{CFD}$ & $1-4.6$ & $0.1-0.25$ & NACA0012, & $2.8-17$ & $2.4-10$ \\
\hline & & & & & NACA0015 & & \\
\hline & Lee and Gerontakos [7] & Experiment & 0.135 & $0.025-0.1$ & NACA0012 & $0-10$ & $5-15$ \\
\hline & Amandolèse and Széchényl [1] & Experiment & 1 & $0.018-0.18$ & NACA $63_{4-}$ & 12 & $2-8$ \\
\hline & & & & & 421 & & \\
\hline & Wang et al. [13] & CFD & $0.135-0.373$ & $0.1-0.15$ & NACA0012 & $10-15$ & $10-15$ \\
\hline & Authors & Method & TSR & $k_{\text {red }}$ & Airfoils & & \\
\hline \multirow{3}{*}{$\begin{array}{l}\text { Rotating } \\
\text { blade }\end{array}$} & Butterfield et al. [6] & Experiment & 2.82 & $0.04-0.12$ & S809 & & \\
\hline & Shipley et al. [10] & Experiment & $1.7-6.3$ & $0.04-0.21$ & S809 & & \\
\hline & Schreck and Robinson [43] & Experiment & $2.5-4.2$ & $0.03-0.26$ & S809 & & \\
\hline
\end{tabular}




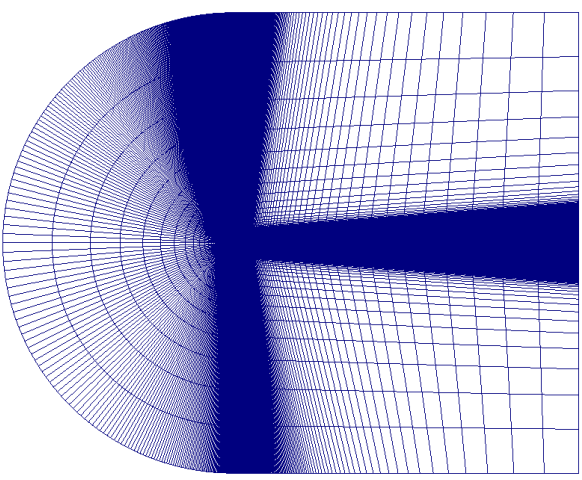

(a)

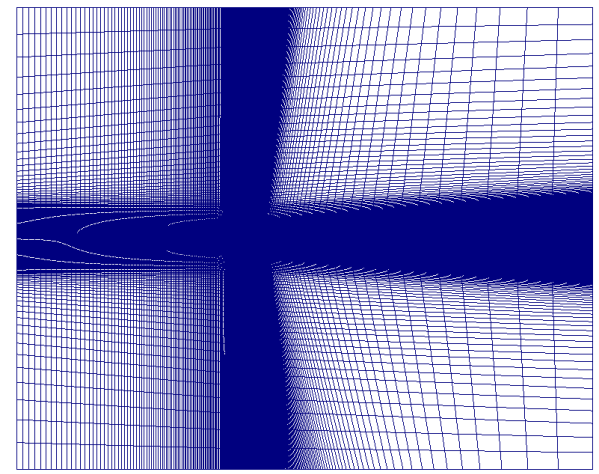

(c)

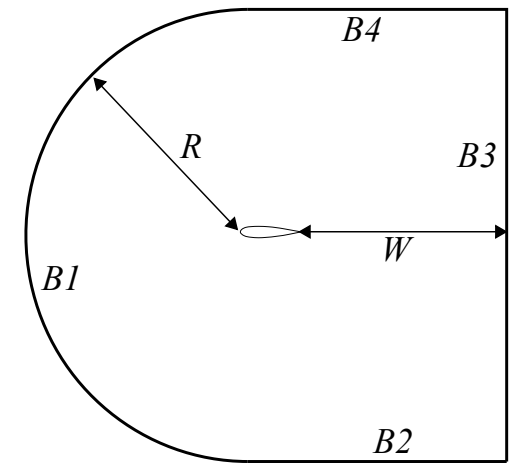

(b)

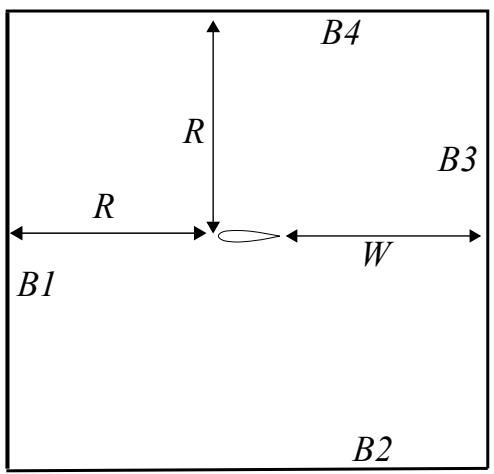

(d)

Figure 1: Mesh topology (a,c) and a sketch of the domain (b,d,not to scale) for a c-type $(\mathrm{a}, \mathrm{b})$ and modified version $(\mathrm{c}, \mathrm{d})$. B1 - B4: the domain boundaries. $R$ : computational domain radius; $W$ : wake length. Also see Tables 2 and 3. 
Table 2: Summary of the boundary conditions. $U_{\infty}$ is the freestream velocity and $d / d n$ is a normal derivative to the boundary. The transverse plane is placed at $x=x_{0}$ where the synthetic turbulence (XCDF [39]) is imposed. See Fig. 1 for the mesh type and boundaries (B1 - B4).

\begin{tabular}{llllll}
\hline \hline Mesh type & B1 & B2 & B3 & B4 & $x_{0} / c=-7$ \\
\hline C-type & $u_{i}=U_{\infty}$, & $u_{i}=U_{\infty}$, & $d u_{i} / d n=0$, & $d u_{i} / d n=0$, & $\mathrm{n} / \mathrm{a}$ \\
& $d p / d n=0$ & $d p / d n=0$ & $p=p_{\infty}$ & $p=p_{\infty}$ & \\
& & & & & \\
Modified & $u_{i}=U_{\infty}$, & $u_{i}=U_{\infty}$, & $d u_{i} / d n=0$, & $d u_{i} / d n=0$, & XCDF [39] \\
& $d p / d n=0$ & $d p / d n=0$ & $p=p_{\infty}$ & $p=p_{\infty}$ & \\
\hline \hline
\end{tabular}

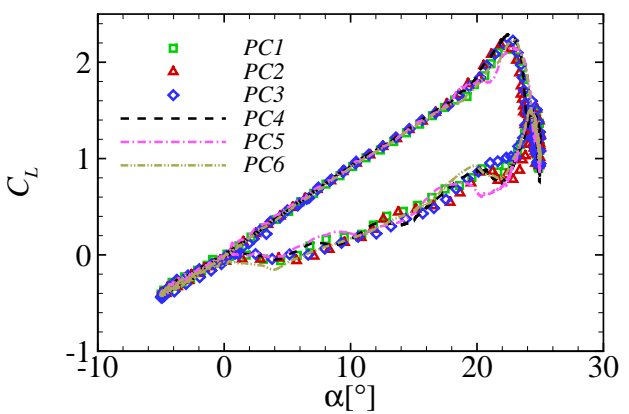

(a)

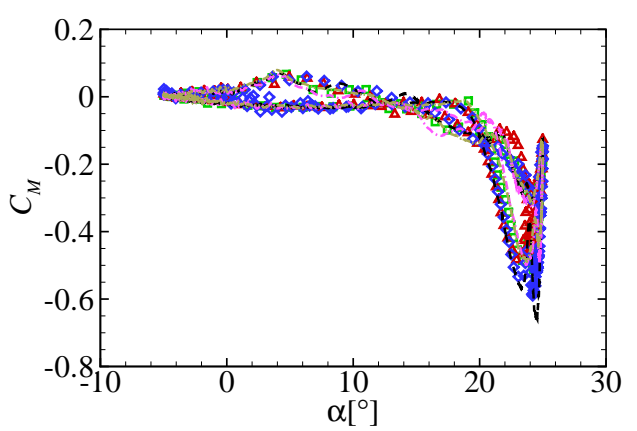

(b)

Figure 2: The effect of resolution and domain size on the lift (a) and moment (b) coefficients for the pitching airfoil at $k_{\text {red }}=0.1$ and $\alpha=10^{\circ}+15^{\circ} \sin (\omega t)$. 
Table 3: The computational domain size in unit $c$ and number of grid points for pitching (PC) airfoils. $R$ : computational domain radius; $W$ : wake length; $L_{z}$ : span length; $N_{R}, N_{W}, N_{z}, N_{\text {low }}$ and $N_{u p}$ : number of grid points per $R, W$ and $L_{z}$, upper airfoil surface and lower airfoil surface, respectively. PC1 - PC6 indicate the case IDs for the pitching (PC) airfoils with different resolutions and the domain width. Also see Fig. 1.

\begin{tabular}{lllllll}
\hline \hline & PC1 & PC2 & PC3 & PC4 & PC5 & PC6 \\
\hline$R[\mathrm{c}]$ & 22 & 22 & 22 & 22 & 22 & 22 \\
$W[\mathrm{c}]$ & 33 & 33 & 33 & 33 & 33 & 33 \\
$L_{z}[\mathrm{c}]$ & 0.5 & 0.5 & 0.5 & 0.5 & 0.5 & 1 \\
\hline$N_{R}$ & 206 & 323 & 206 & 206 & 206 & 206 \\
$N_{W}$ & 66 & 66 & 81 & 81 & 81 & 81 \\
$N_{\text {up }}$ & 386 & 386 & 700 & 386 & 386 & 386 \\
$N_{\text {low }}$ & 193 & 193 & 193 & 193 & 193 & 193 \\
$N_{z}$ & 40 & 40 & 40 & 80 & 20 & 80 \\
\hline \hline
\end{tabular}




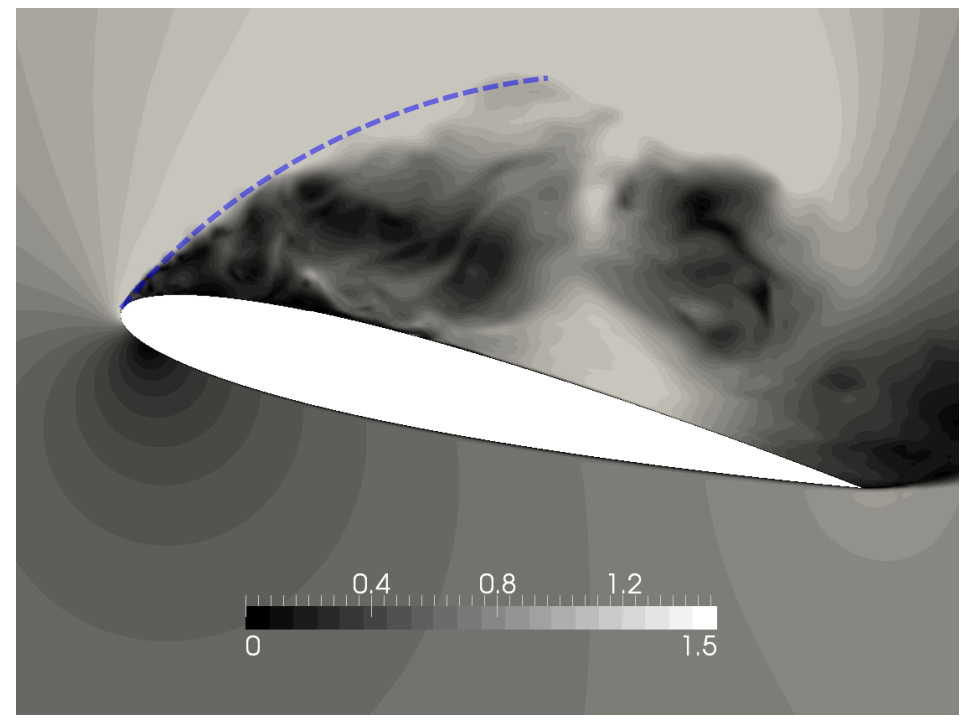

Figure 3: A snapshot of the velocity magnitude normalized by $U_{\infty}$ for case PC5 at $k_{\text {red }}=$ 0.1 and $\alpha=22.9^{\circ} \uparrow$. The dashed-line indicates the edge of the free shear layer near the leading edge.

Table 4: The effect of the reduced frequency on crucial unsteady aerodynamic data. $\alpha_{L, \max }$ is the angle of attack where the maximum lift occurs.

\begin{tabular}{llllll}
\hline \hline Case & $k_{\text {red }}$ & $C_{L, \max }$ & $C_{M, \min }$ & $C_{D, \max }$ & $\alpha_{L, \max }$ \\
\hline $\operatorname{Exp}[7]$ & 0.025 & 1.47 & -0.143 & 0.425 & $17.5^{\circ}$ \\
$\operatorname{Exp}[7]$ & 0.05 & 1.87 & -0.211 & 0.66 & $21.1^{\circ}$ \\
$\operatorname{Exp}[7]$ & 0.1 & 2.44 & -0.263 & 0.91 & $24.7^{\circ}$ \\
\hline LES2 & 0.025 & 1.49 & -0.159 & 0.412 & $16.4^{\circ}$ \\
LES2 & 0.05 & 1.74 & -0.287 & 0.629 & $19.5^{\circ}$ \\
LES2 & 0.1 & 2.01 & -0.345 & 0.856 & $22.8^{\circ}$ \\
\hline \hline
\end{tabular}




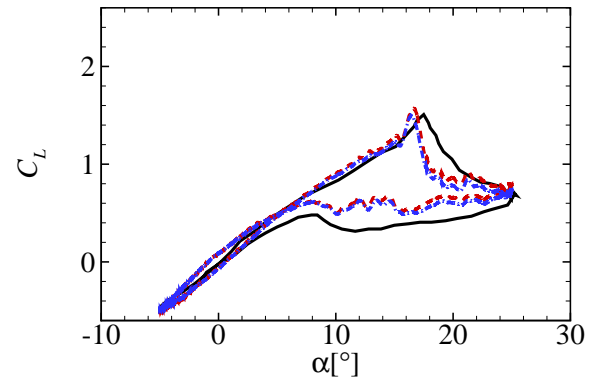

(a) $k_{\text {red }}=0.025$

ev

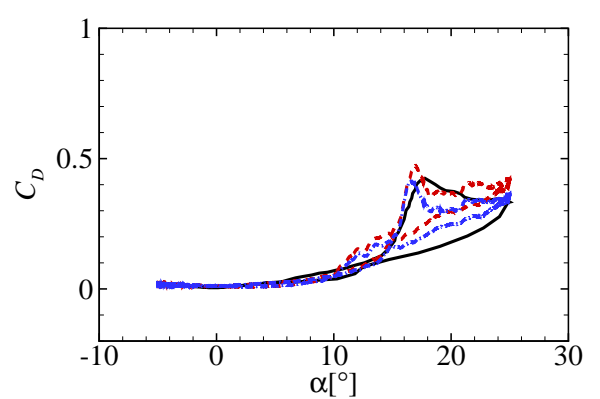

(d) $k_{\text {red }}=0.025$

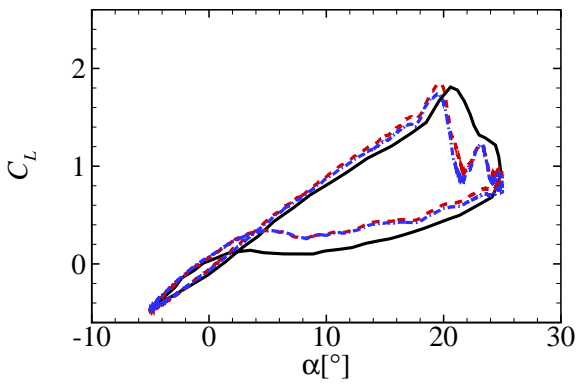

(b) $k_{\text {red }}=0.05$

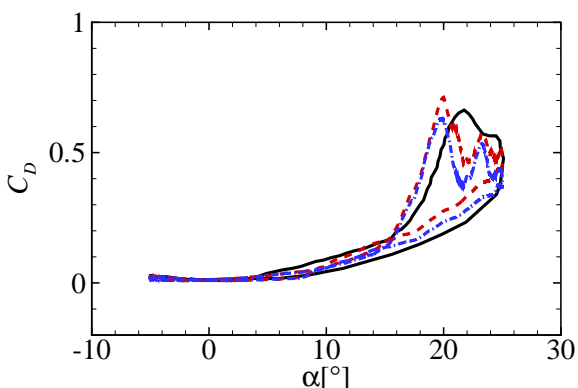

(e) $k_{\text {red }}=0.05$

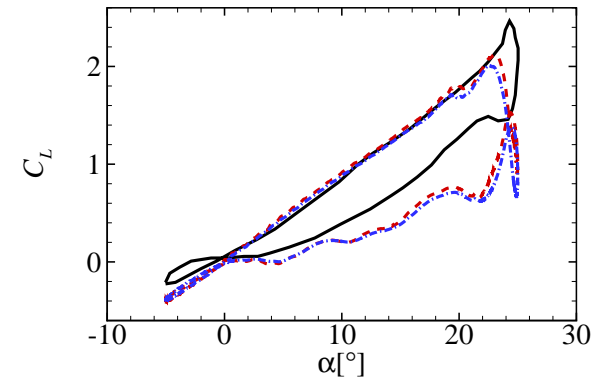

(c) $k_{\text {red }}=0.1$

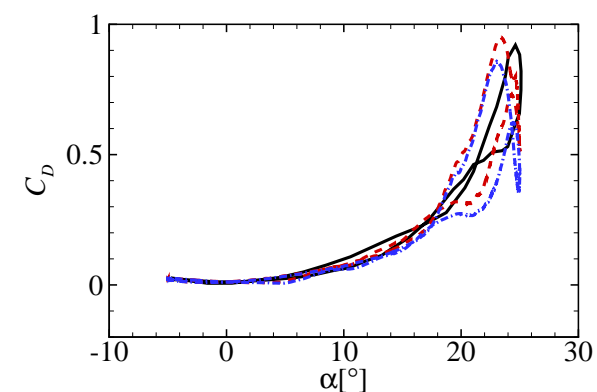

(f) $k_{\text {red }}=0.1$

Figure 4: Phase-averaged lift and drag coefficients. — Exp [7], - - LES1, -- LES2. LES1: aerodynamic forces obtained by integrating over the entire airfoil surface; LES2, over $80 \%$ only of the airfoil surface from the leading edge. Both LES1 and LES2 are based on the mesh for case PC5. 


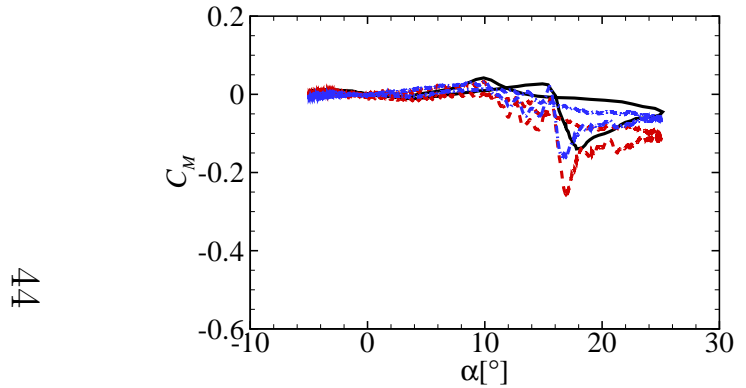

(a) $k_{\text {red }}=0.025$

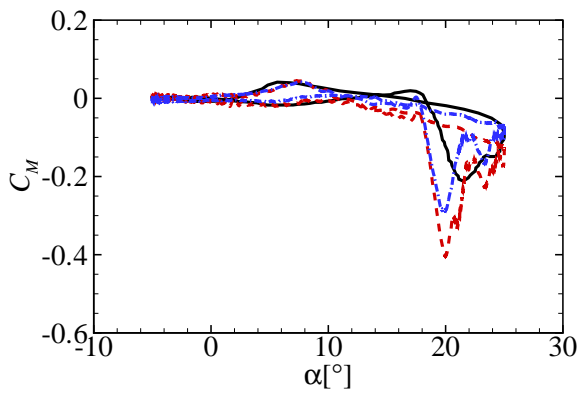

(b) $k_{\text {red }}=0.05$

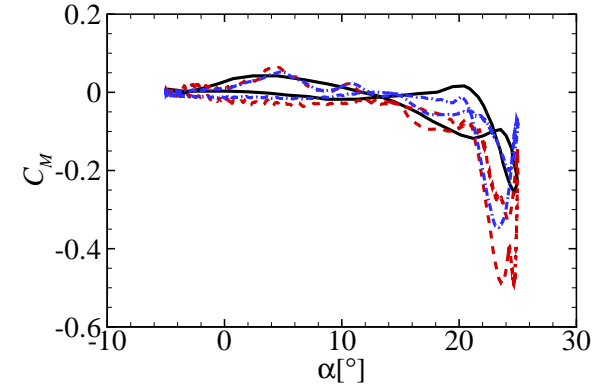

(c) $k_{\text {red }}=0.1$

Figure 5: As Fig. 4, but moment coeffient. 


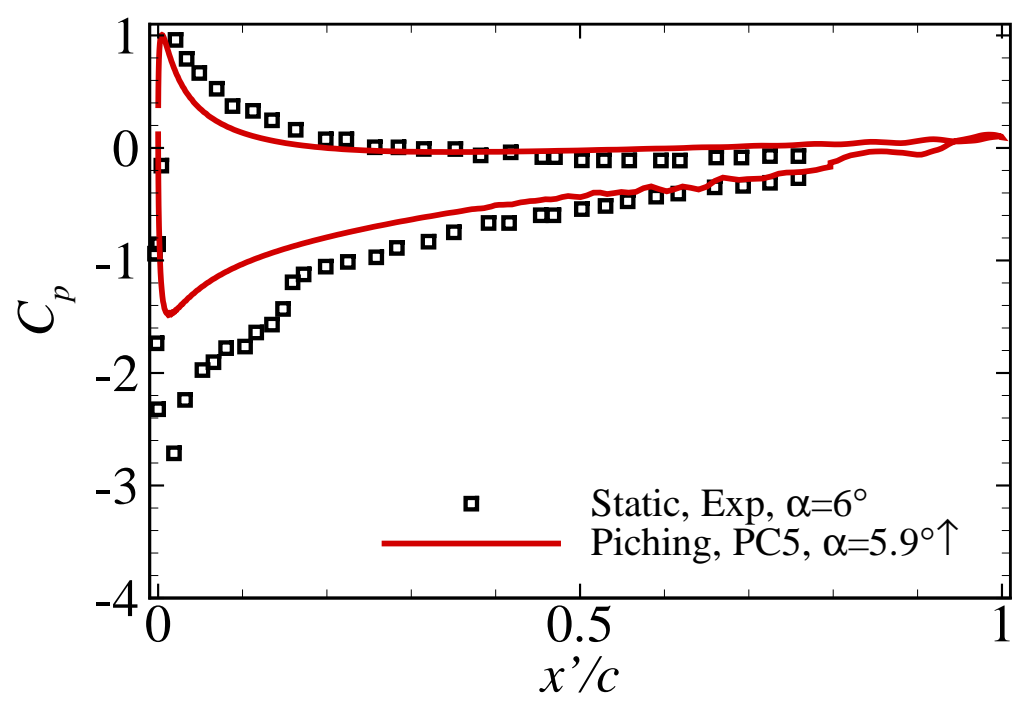

(a)

Figure 6: Pressure coefficient distribution. LES data, case PC5 at $\alpha=5.9^{\circ} \uparrow$ and $k_{\text {red }}=$ 0.05. Experimental data [7], a static airfoil at $\alpha=6^{\circ}$ with the same airfoil and Reynolds number. 

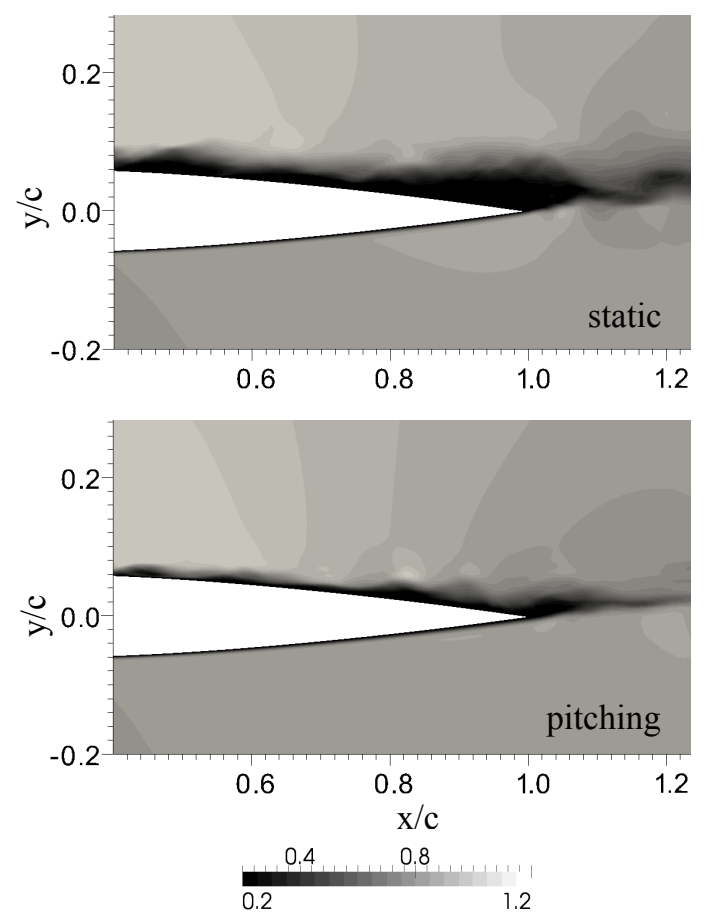

Figure 7: Instantaneous velocity magnitude contour near the trailing edge for the static (top: the PC5 mesh, $\alpha=10^{\circ}$ ) and pitching (bottom: PC5, $\alpha=10.1^{\circ} \uparrow, k_{\mathrm{red}}=0.05$ ) airfoils at the middle section of the span. The velocity contour is normalized by $U_{\infty}$.

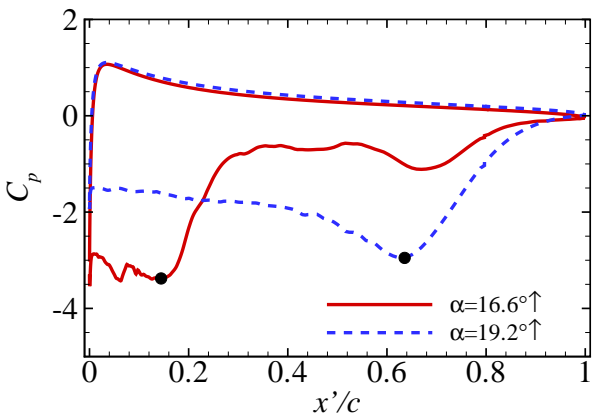

(a)

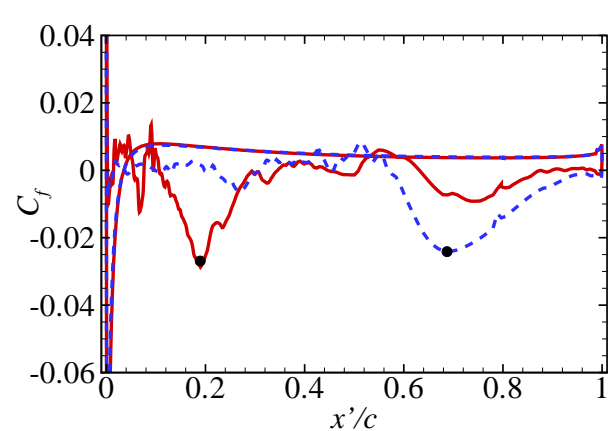

(b)

Figure 8: (a) Spanwise-averaged pressure and (b) skin-friction coefficients at $\alpha=16.6^{\circ} \uparrow$ and $19.2^{\circ} \uparrow$ during the upstroke. $k_{\text {red }}=0.05$; mesh, case PC5. 

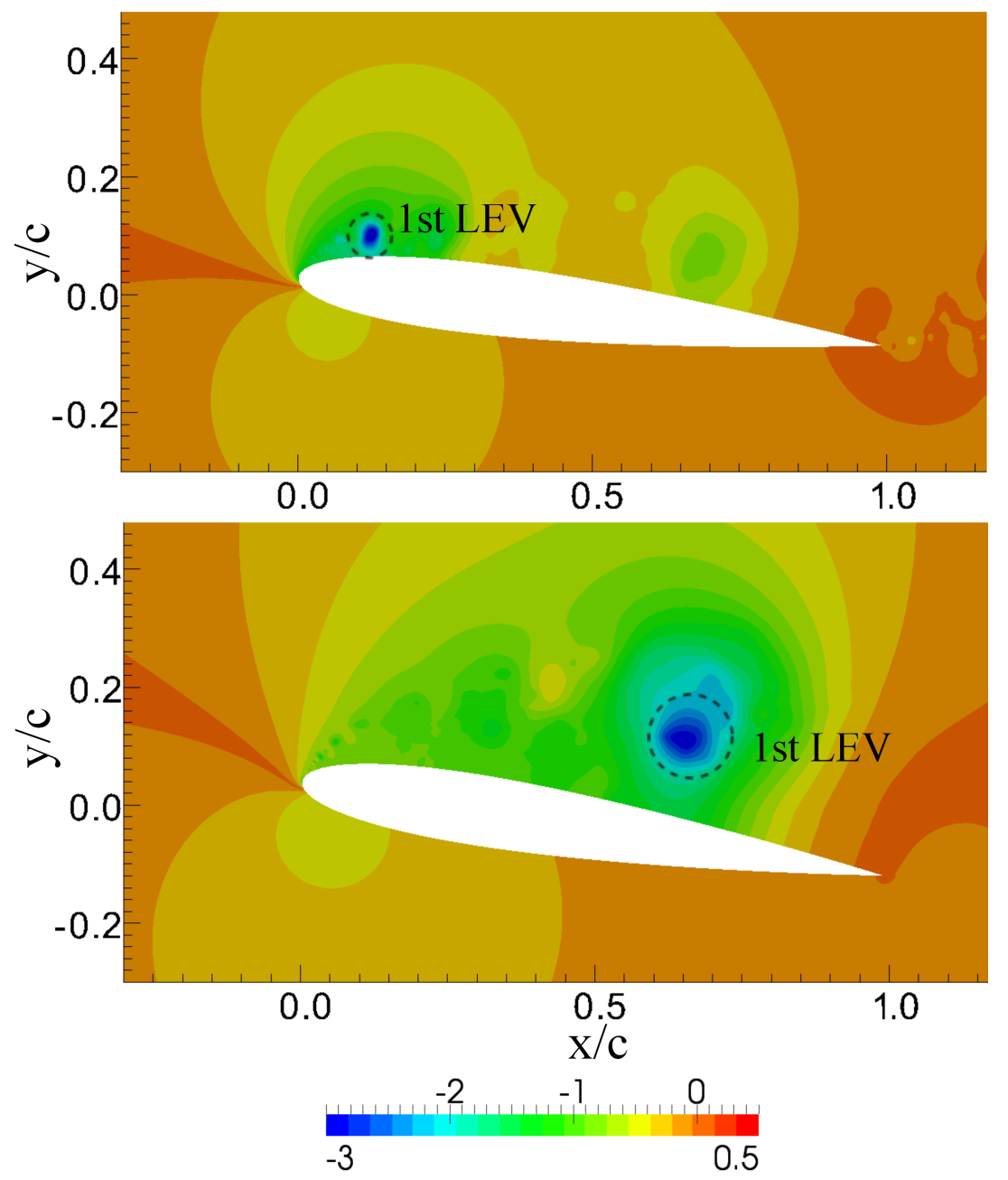

Figure 9: Negative pressure peaks normalized by $\rho U_{\infty}^{2}$ over the airfoil. $k_{\text {red }}=0.05$; PC5 mesh; $\alpha=16.6^{\circ} \uparrow$ (top), 19.2 $\uparrow$ (bottom). 


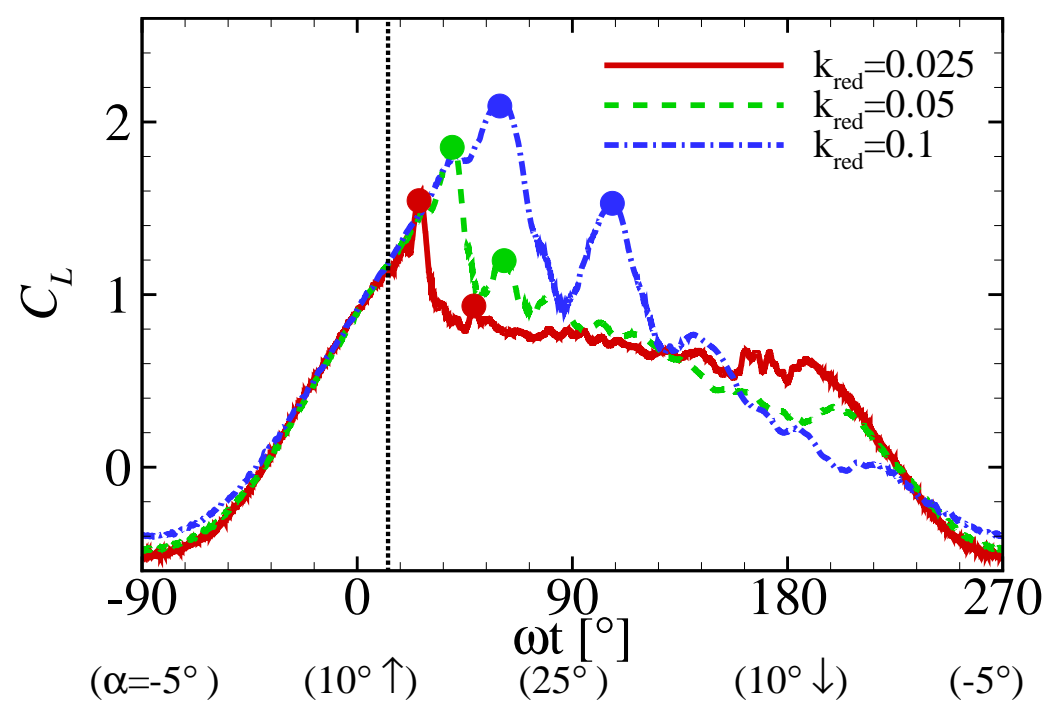

Figure 10: Lift coefficients (from LES2 in Fig. 4) versus phase angles at different reduced frequencies $k_{\text {red. }}$. The large dots indicate the lift peaks due to the shedding of the leading edge vortices. The vertical dot-line indicates the static stall angle, i.e. $\alpha=13^{\circ}[7]$. 


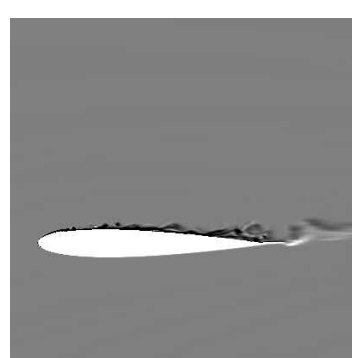

(a) $1 . \alpha=10^{\circ} \uparrow$

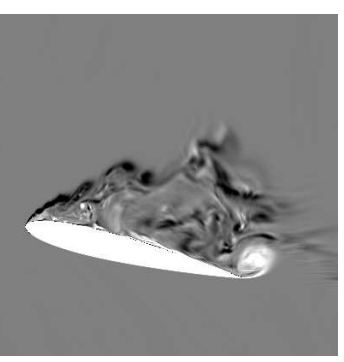

(f) $6 . \alpha=23.3^{\circ} \uparrow$

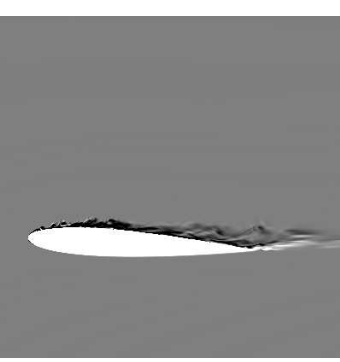

(b) $2 . \alpha=13^{\circ} \uparrow$

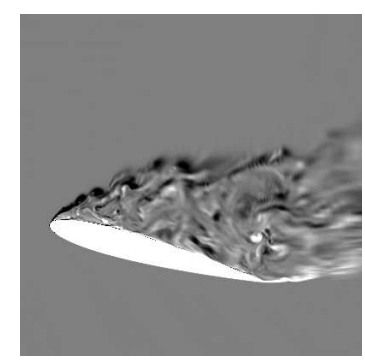

(g) 7. $\alpha=24.8^{\circ} \uparrow$

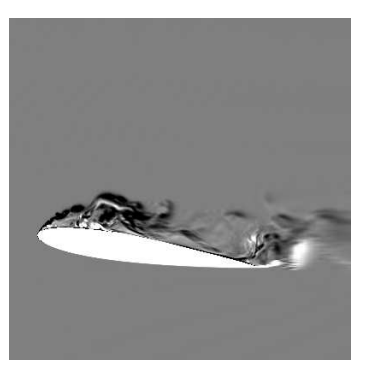

(c) $3 . \alpha=18.2^{\circ} \uparrow$

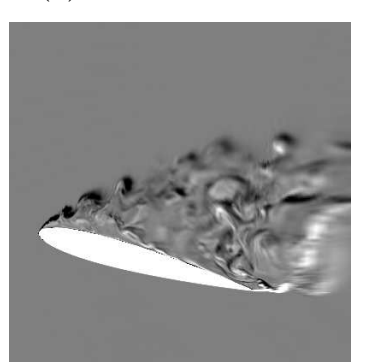

(h) $8 . \alpha=25^{\circ}$

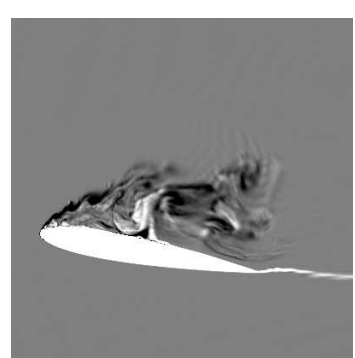

(d) $4 . \alpha=19.9^{\circ} \uparrow$

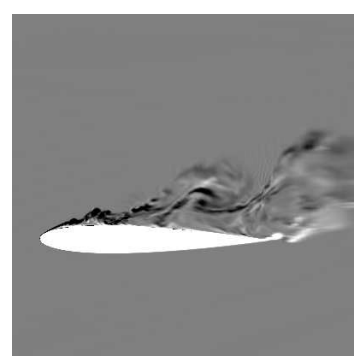

(i) $9 . \alpha=10.2^{\circ} \downarrow$

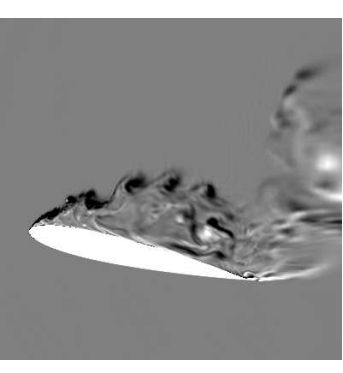

(e) $5 . \alpha=22.4^{\circ} \uparrow$

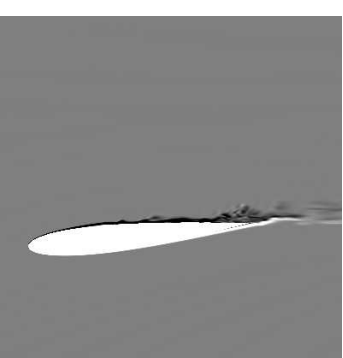

(j) 10. $\alpha=4^{\circ} \downarrow$

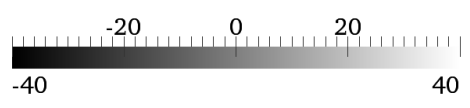

Figure 11: The instantaneous $z$-component of vorticity normalized by $c$ and $U_{\infty}$ at the middle section of the span. $k_{\text {red }}=0.05$. Note that the chord line is aligned to the $x$-axis at $\alpha=10^{\circ}$ as the angle of attack is realised by the velocity components at the boundaries, i.e. $u=U_{\infty} \cos \left(10^{\circ}\right)$ and $v=U_{\infty} \sin \left(10^{\circ}\right)$. 


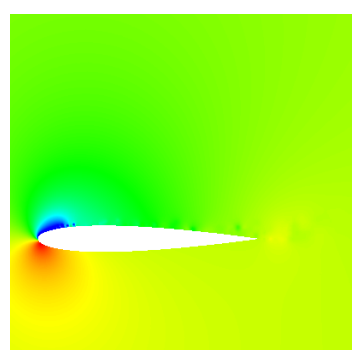

(a) $1 . \alpha=10^{\circ} \uparrow$

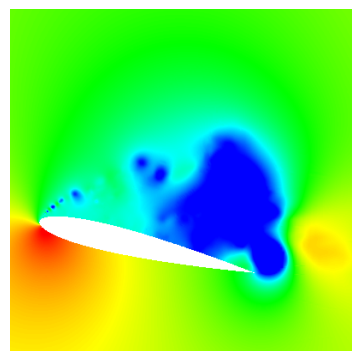

(f) $6 . \alpha=23.3^{\circ} \uparrow$

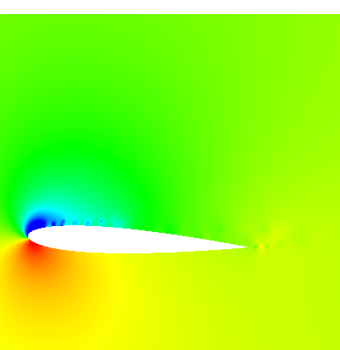

(b) $2 . \alpha=13^{\circ}$

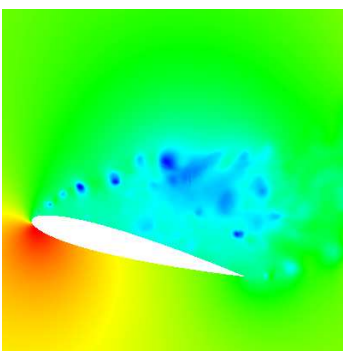

(g) 7. $\alpha=24.8^{\circ} \uparrow$

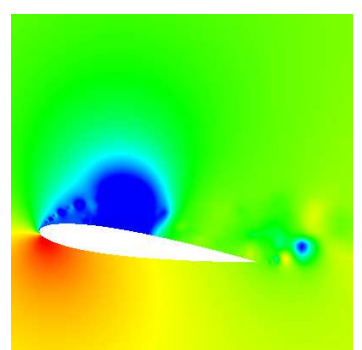

(c) 3. $\alpha=18.2^{\circ} \uparrow$

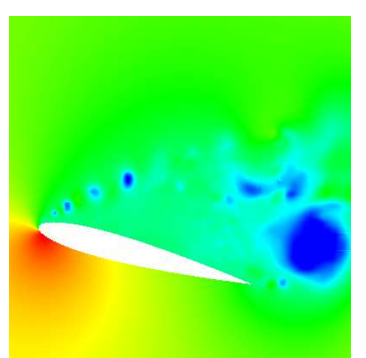

(h) $8 . \alpha=25^{\circ}$

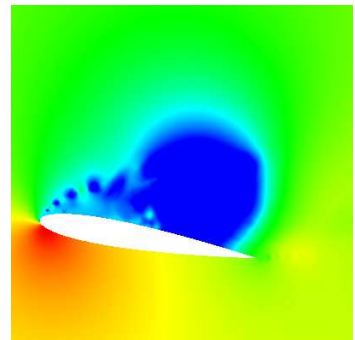

(d) 4. $\alpha=19.9^{\circ} \uparrow$

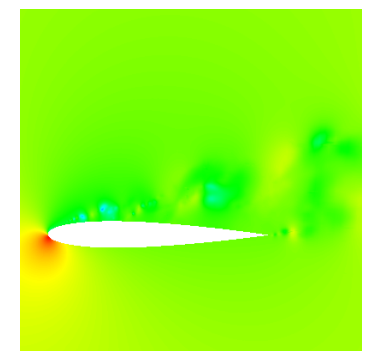

(i) $9 . \alpha=10.2^{\circ}$

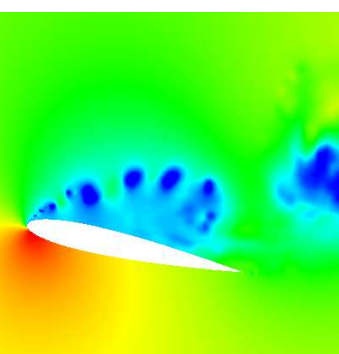

(e) $5 . \alpha=22.4^{\circ} \uparrow$

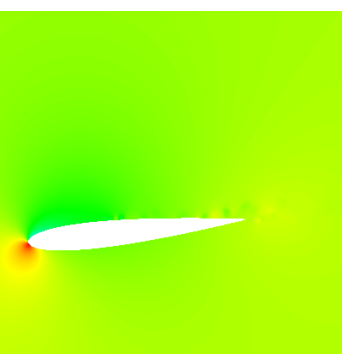

(j) $10 . \alpha=4^{\circ} \downarrow$

$$
\begin{array}{rr}
-0.8 & -0.4 \quad 0.4 \\
-1 & 0.5
\end{array}
$$

Figure 12: The instantaneous pressure contours normalized by $\rho U_{\infty}^{2}$ at the middle section of the span. $k_{\text {red }}=0.05$. 


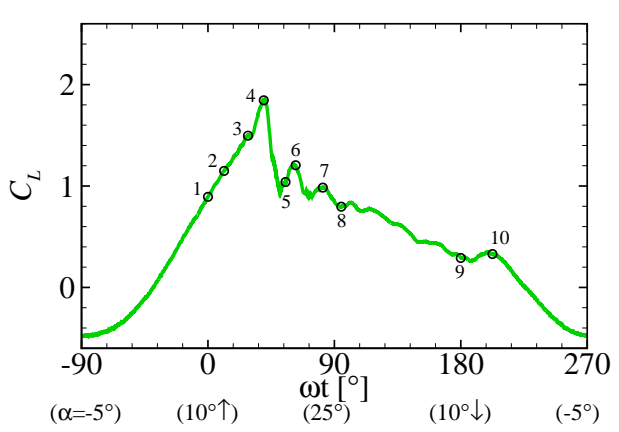

(a)

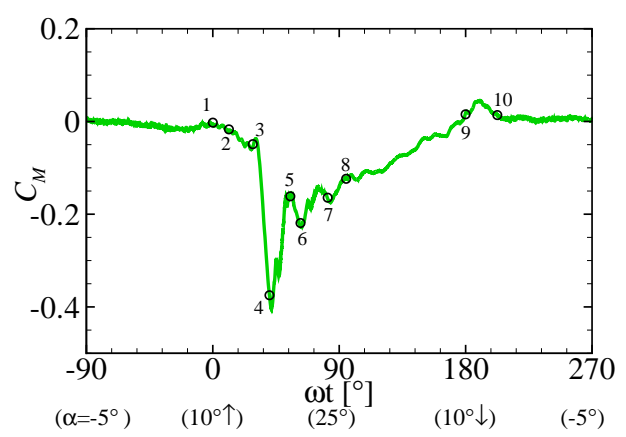

(b)

Figure 13: (a) Lift and (b) moment coefficients (from LES2 in Fig. 4) versus phase angles. $k_{\text {red }}=0.05$. The numbers marked on the curves correspond with the snapshots in Figs. 11 and 12. 1: $\alpha=10^{\circ} \uparrow, 2: \alpha=13^{\circ} \uparrow, 3: \alpha=18.2^{\circ} \uparrow, 4: \alpha=19.9^{\circ} \uparrow, 5: \alpha=22.4^{\circ} \uparrow, 6:$ $\alpha=23.3^{\circ} \uparrow, 7: \alpha=24.8^{\circ} \uparrow, 8: \alpha=25^{\circ}, 9: \alpha=10.2^{\circ} \downarrow, 10: \alpha=4^{\circ} \downarrow$.

Table 5: Turbulence intensity (TI), integral length scales $\left(I_{i j}\right)$ and Reynolds number for the domain with the airfoil removed. The inflow is generated at $x / I_{11}=-23.3$ and the airfoil will be placed at $x / I_{11}=0 . R e_{I}=U_{\infty} I_{11} / \nu, \operatorname{Re}_{\lambda}=\left(6.7 R e_{I}\right)^{1 / 2}[44] . I_{11}=I_{21}=I_{31}$, $I_{i 2}=I_{i 3}=0.5 I_{i 1}$ where $i=1,2,3$.

\begin{tabular}{lllll}
\hline \hline$x / I_{11}$ & $\mathrm{TI}[\%]$ & $I_{11} / c$ & $R e_{I}$ & $R e_{\lambda}$ \\
\hline-23.3 & 5 & 0.3 & 40,500 & 520 \\
0 & 4.5 & 0.43 & 58,050 & 622 \\
\hline-23.3 & 10 & 0.3 & 40,500 & 520 \\
0 & 6.3 & 0.47 & 64,350 & 650 \\
\hline \hline
\end{tabular}




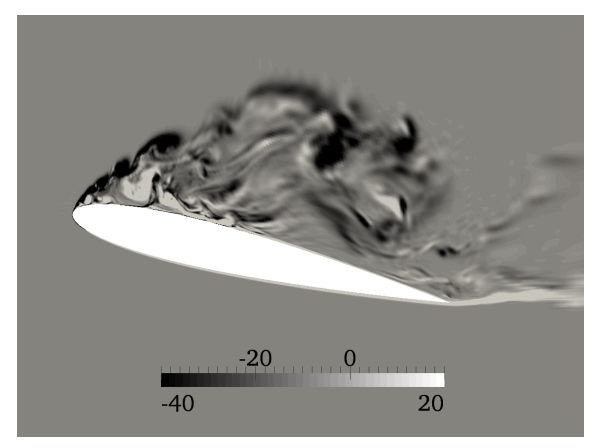

(a) z-vorticity

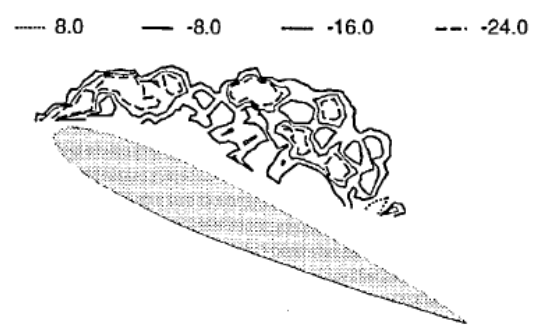

(c) z-vorticity

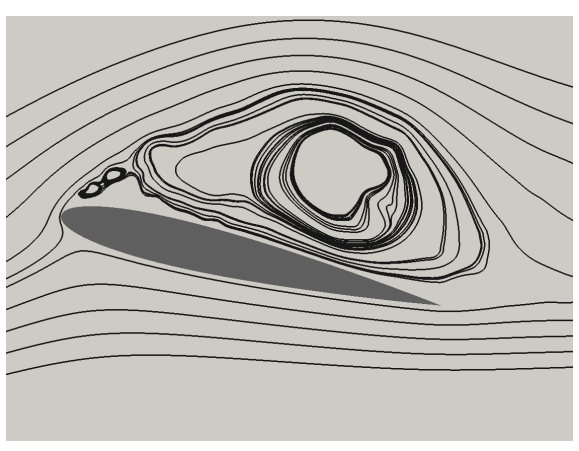

(b) Streamlines

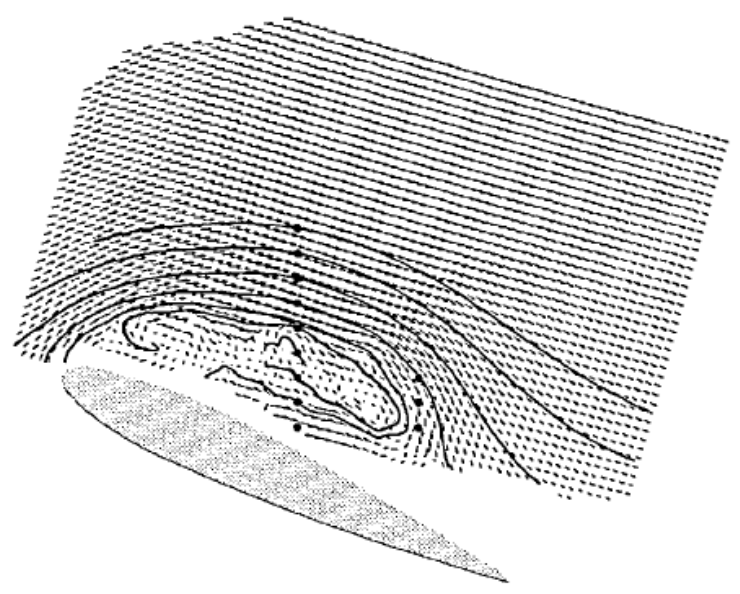

(d) velocity vector and streamline

Figure 14: Snapshots of the instantaneous flows over a pitching NACA 0012 airfoil at the middle section of the span. (a) and (b): LES, PC5 mesh, at $\alpha=23.3^{\circ} \uparrow(\alpha(t)=$ $\left.10^{\circ}+15^{\circ} \sin (\omega t)\right), R e=135,000, k_{\text {red }}=0.1 ;$ (c) and (d): experiment [36] at $\alpha=24^{\circ} \uparrow($ $\left.\alpha(t)=15^{\circ}+10^{\circ} \sin (\omega t)\right), R e=373,000, k_{\text {red }}=0.15$. 


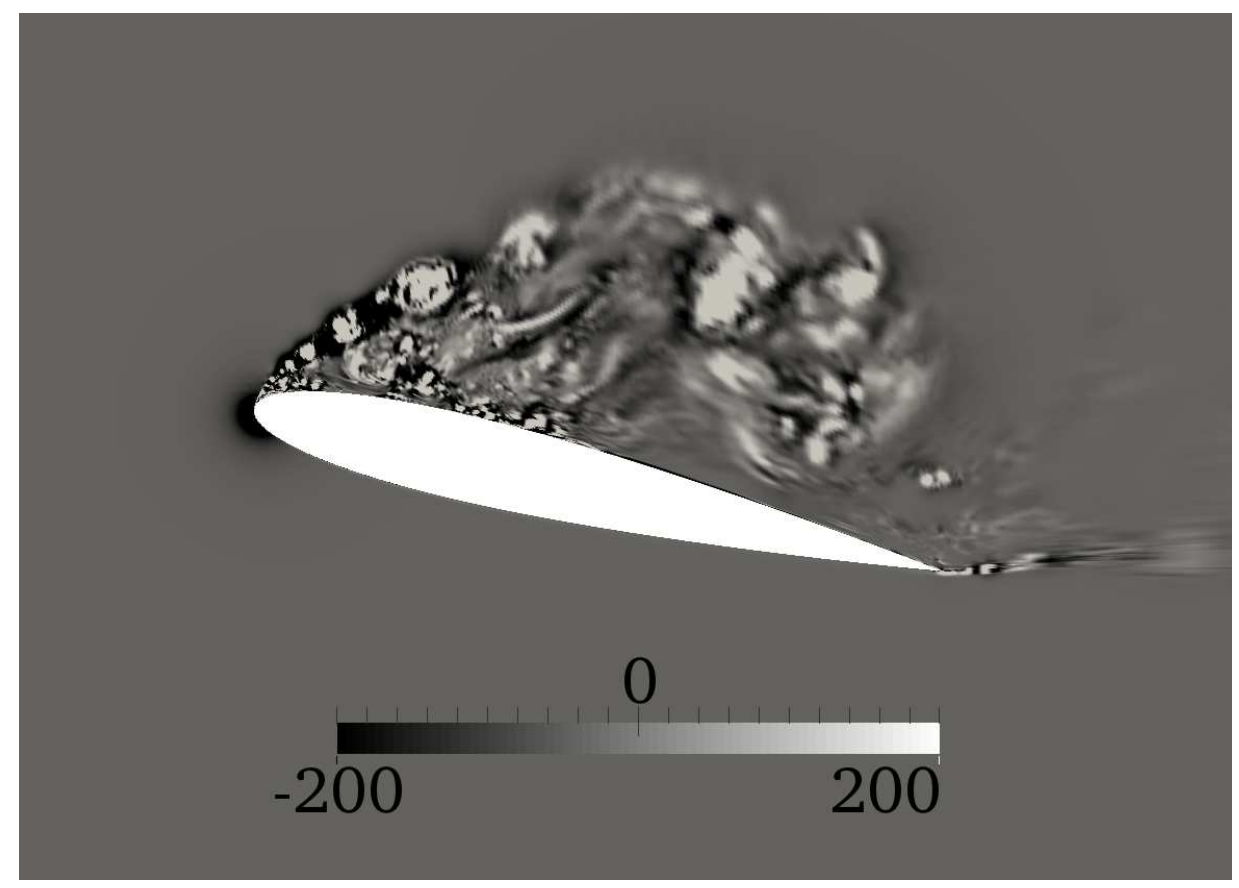

Figure 15: Instantaneous iso-contours of a vortex identifier $\lambda_{2}$ normalized by chord length $c$ and freestream velocity $U_{\infty}$ for case PC5 at $k_{\text {red }}=0.1, \alpha=23.3^{\circ} \uparrow$.

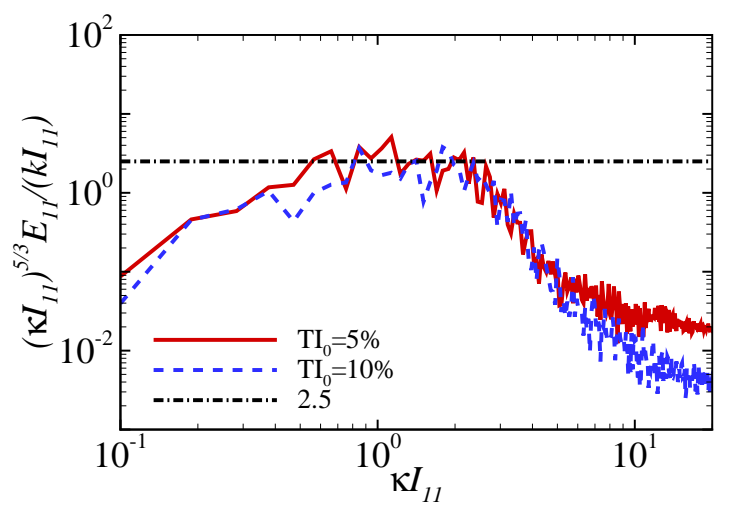

Figure 16: One-dimensional compensated energy spectra $E_{11}$ of the streamwise velocity component normalized by the local turbulent kinetic energy $k$ at $x / I_{11}=23.3$ (see Table 5). The dot-dashed line is the inertial region value, 2.5 . 


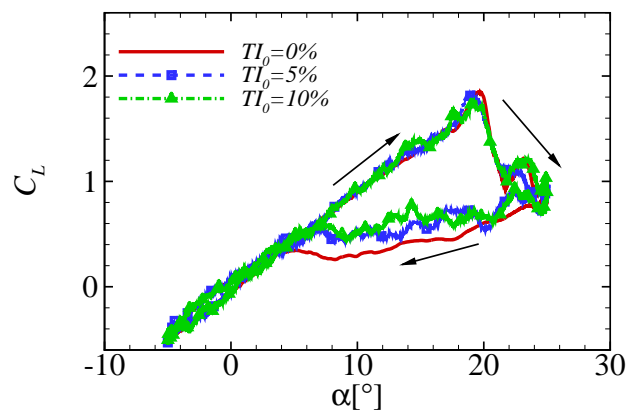

(a)

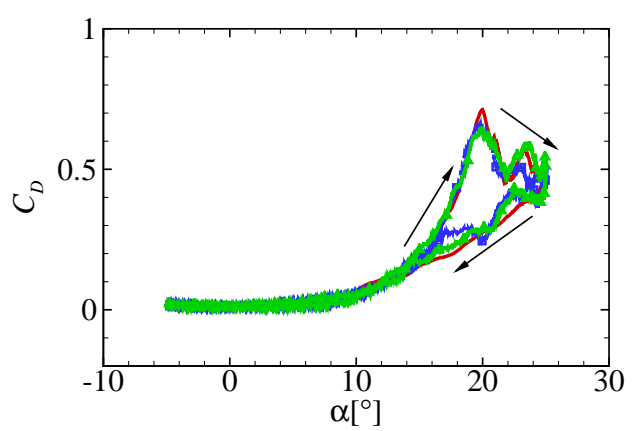

(b)

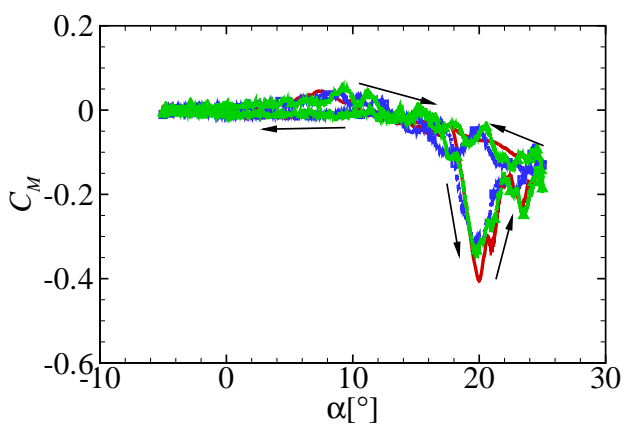

(c)

Figure 17: Effect of freestream turbulence on lift, drag and moment coefficients. $k_{\text {red }}=$ 0.05. PC5 mesh. The effective turbulence intensities at the leading edge are $4.5 \%$ and $6.3 \%$ for $T I_{0}=5 \%$ and $10 \%$ respectively (Table 5 ). 


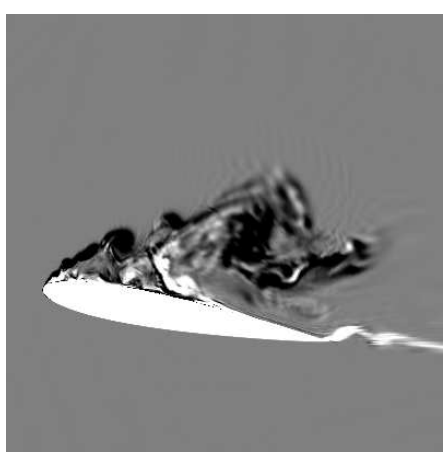

(a) $T I_{0}=0 \%, \alpha=20.3 \uparrow$

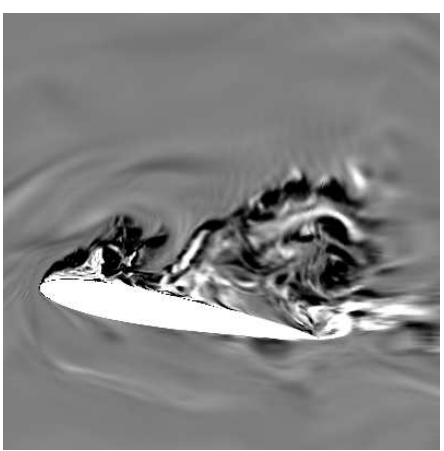

(e) $T I_{0}=10 \%, \alpha=20.3 \uparrow$

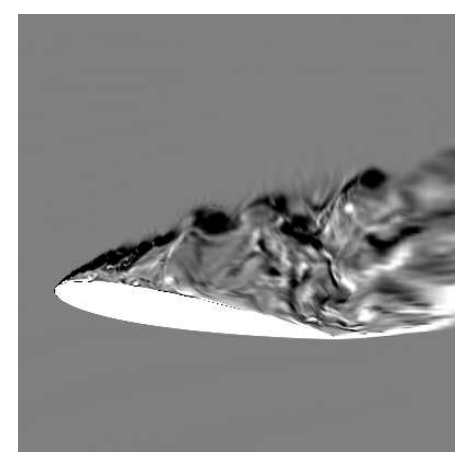

(b) $T I_{0}=0 \%, \alpha=19.5 \downarrow$

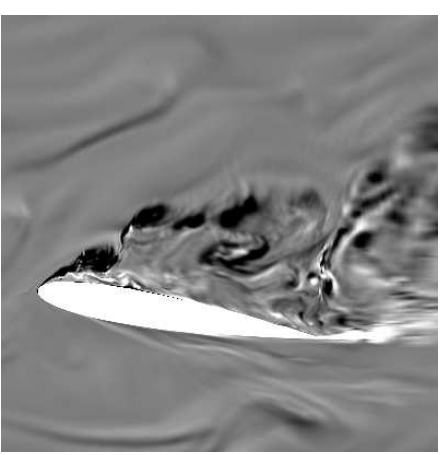

(f) $T I_{0}=10 \%, \alpha=19.5$

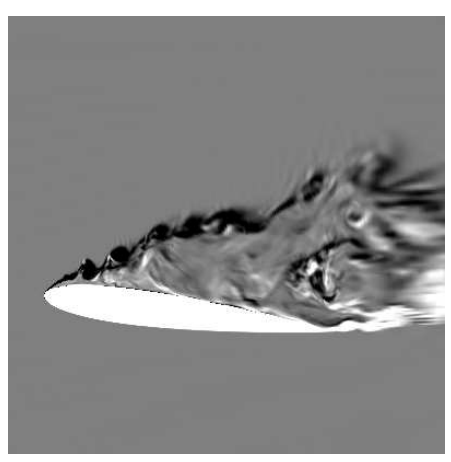

(c) $T I_{0}=0 \%, \alpha=17 \downarrow$

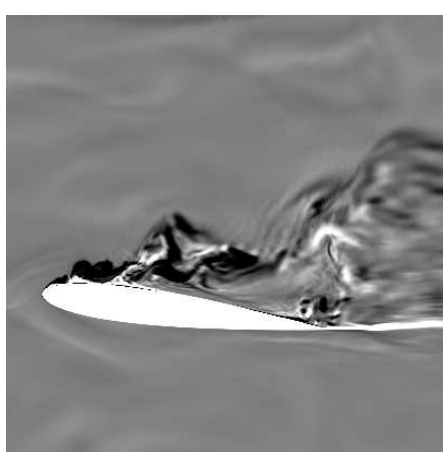

(g) $T I_{0}=10 \%, \alpha=17 \downarrow$ $\begin{array}{rrrr} & -10 & 0 & 10 \\ -20 & & 10\end{array}$

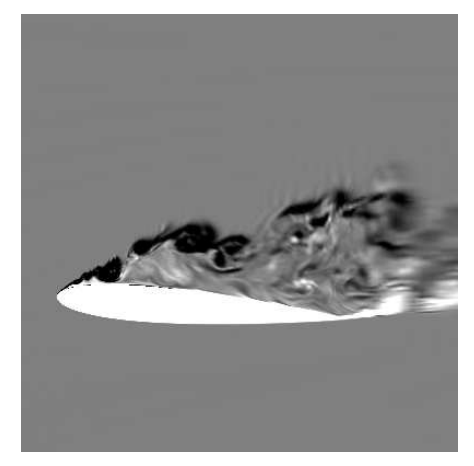

(d) $T I_{0}=0 \%, \alpha=14.2 \downarrow$

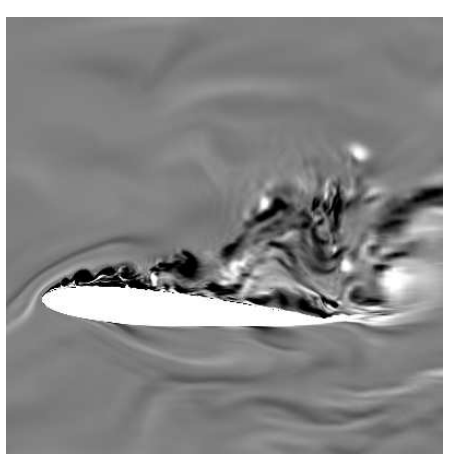

(h) $T I_{0}=10 \%, \alpha=14.2 \downarrow$

Figure 18: A comparison of instantaneous z-vorticity contours at the mid-span for $T I_{0}=0 \%$ (top-row) and $T I_{0}=10 \%$ (bottom-row). Vorticity normalized by $U_{\infty}$ and $c$. 


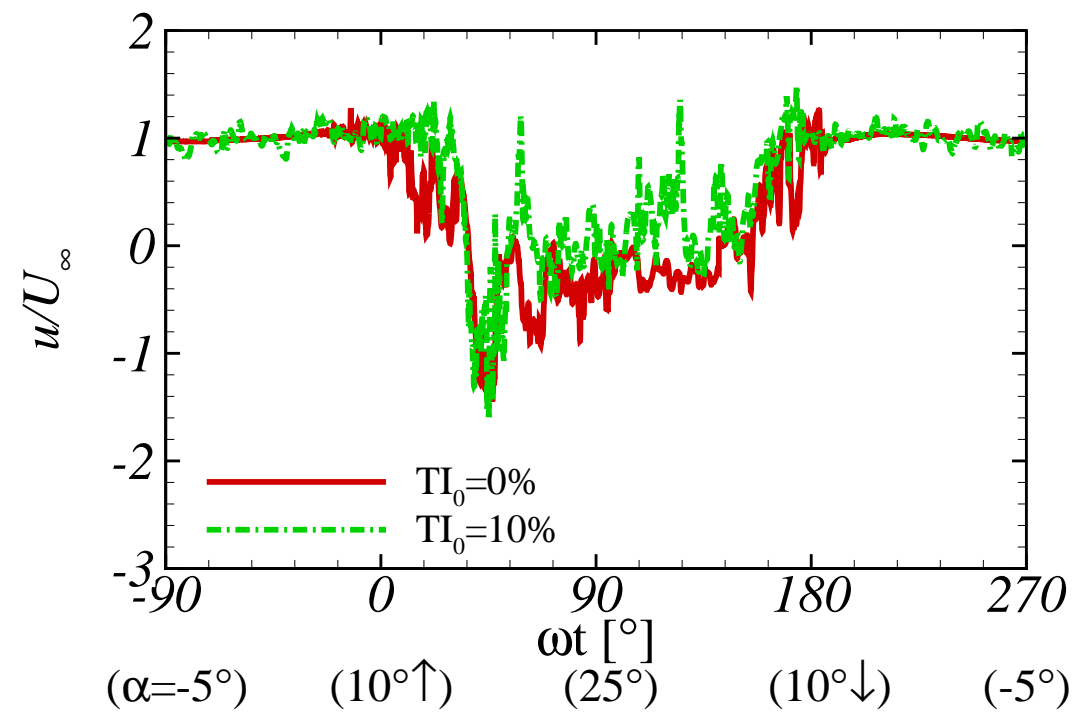

(a)

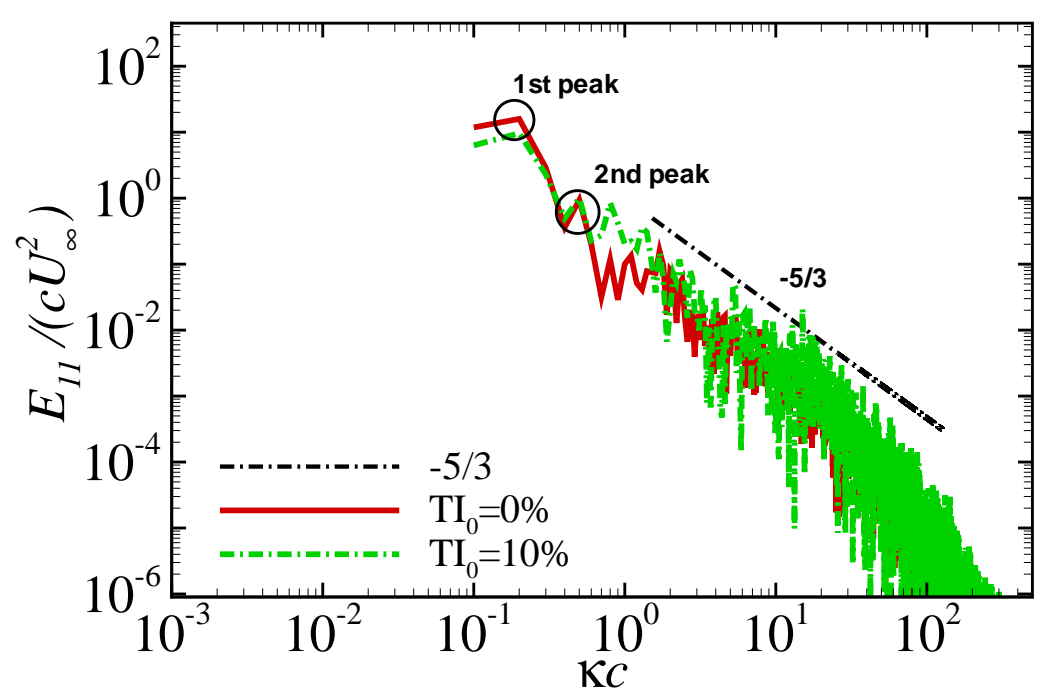

(b)

Figure 19: Time series of the instantaneous streamwise velocity sampled at $x / c=0.75$ and $y / c=0.2$ for one cycle duration (a) and their energy spectra (b). $T I_{0}=0,10 \%$. 